\title{
Monetary Equilibria and Knightian Uncertainty
}

\author{
Eisei Ohtaki ${ }^{\dagger} \quad$ Hiroyuki Ozaki ${ }^{\ddagger}$
}

$\dagger$ Faculty of Economics, Kanagawa University, 3-27-1 Rokkakubashi, Kanagawa-ku, Yokohama-shi, Kanagawa 221-8686, Japan

Email address: ohtaki@kanagawa-u.ac.jp

$¥$ Faculty of Economics, Keio University, 2-15-45 Mita, Minato-ku, Tokyo 108-8345, Japan

Email address: ozaki@econ.keio.ac.jp

Current Draft: December 7, 2014

\begin{abstract}
This paper considers a pure-endowment stationary stochastic overlapping generations economy, in which agents have maxmin expected utility preferences. Two main results are obtained. First, we show that multiple stationary monetary equilibria exist. Consequently, real and price indeterminacies arise under the assumption that aggregate shock exists. Second, we show that each of these stationary monetary equilibria is conditionally Pareto optimal; i.e., no other stationary allocations strictly Pareto dominate the equilibrium allocations.
\end{abstract}

Keywords: Money, Maxmin expected utility, Conditional Pareto optimality, Indeterminacy, Stochastic overlapping generations model

JEL Classification Numbers: E40, D81, D50, D61. 


\section{Introduction}

Since Savage's (1954) pioneering work, most studies on economics under uncertainty have considered agents who choose their actions to maximize the expected utility with a unique prior. Ellsberg's (1961) example, however, highlighted a plausible situation where agents may not have a belief about the uncertain situation that is summarized by a single prior because they have only too imprecise information. Methods for explaining Ellsberg's example of decision making under imprecise information did not appear until 20 years later, in the seminal papers by Schmeidler $(1982,1989)$ and Gilboa and Schmeidler (1989). They axiomatized the maxmin expected utility (MEU) preference, under which agents attempt to maximize the expected utility minimized over a set of priors, not a unique prior. ${ }^{1}$ Although the class of preferences with multiple priors includes the class of expected utility preferences with a unique prior as a special case, the situation where an agent's belief is summarized by a set of priors (and not by a unique prior) is now called ambiguity. ${ }^{2}$

This remarkable advance in decision theory has been welcomed in a broad range of disciplines including economics, game theory and finance. ${ }^{3}$ In the last two decades, implications of ambiguity have been explored in several general equilibrium settings. As shown by Dow and Werlang (1992), Tallon (1998), Chateauneuf, Dana and Tallon (2000) and Dana (2004), one of the most important findings in the existing literature is that indeterminacy of equilibria can be generated in the static Arrow-Debreu economy under ambiguity when there is no aggregate uncertainty. ${ }^{4}$ Here, no aggregate uncertainty means that the total endowment does not change over the state space. ${ }^{5}$ However, Rigotti and Shannon (2012) argued that such indeterminacy

\footnotetext{
${ }^{1}$ To be more precise, axiomatizations of the MEU preference by Schmeidler $(1982,1989)$ and Gilboa and Schmeidler (1989) were demonstrated in the framework of Anscombe and Aumann (1963), not in Savage's. Furthermore, Schmeidler (1989) a xiomatized the Choquet expected utility (CEU) preference and showed the class of convex CEU preferences belongs to the class of MEU preferences. By relaxing Savage's postulates, the MEU preference was axiomatized in Savage's framework by Casadesus-Masanell, Klibanoff and Ozdenoren (2000) and Alon and Schmeidler (2014).

${ }^{2}$ According to the terminologies proposed by Knight (1921), the situation where the agents' beliefs are not summarized by a single probability measure is called Knightian uncertainty. Thus, ambiguity is a special case of Knightian uncertainty, and the MEU preference is a special case of ambiguity.

${ }^{3}$ There are good literature surveys such as Epstien and Shneider (2010), Gilboa and Marinacci (2013) and Guidolin and Rinaldi (2013). Etner, Jeleva and Tallon (2012) surveyed an ever growing literature regarding decision theory under ambiguity after the papers by Schmeidler (1989) and Gilboa and Schmeidler (1989) and they also considered applications of ambiguity to economics.

${ }^{4}$ See also Billot, Chateauneuf, Gilboa and Tallon (2000).

${ }^{5}$ Epstein and Wang (1994) use similar logic to no aggregate uncertainty in order to show indeterminacy of equi-
} 
is not robust in the canonical Arrow-Debreu economy; i.e., the equilibrium is generically determinate. Roughly speaking, this means that indeterminacy would disappear if the economy was "shaken" only a little bit. In fact, the set of Arrow-Debreu economies with no aggregate uncertainty has zero Lebesgue measure. This suggests the importance of examining what kind of mechanisms can generate robust equilibrium indeterminacy under ambiguity by embedding it into the general equilibrium framework. The main subject of this paper is to present such a mechanism by embedding ambiguity into a stochastic overlapping generations (OLG) economy.

The OLG model is an intertemporal general equilibrium model that has a demographic structure with overlapping generations. Although the OLG model can be considered at various levels of generality, this paper emphasizes the role of ambiguity by considering a simple but rather canonical stationary pure-endowment stochastic OLG model. Time is divided into discrete dates and runs from 1 to infinity. At each date, one of the finite states is realized. A history of states is called a "date-event." At each date-event, a single perishable commodity is available and a new agent that lives for two consecutive dates is born. Each agent has the opportunity to trade a single infinitely-lived outside asset (money), which has a constant supply and yields no dividend. There exists neither storage technology nor production. Therefore, the ingredients of our model are almost the same as those found in a canonical stochastic OLG model. A remarkable departure of this paper from the standard stochastic OLG model is that each agent is endowed with the MEU preference.

This paper will make four main achievements. Firstly, we show that a stationary monetary equilibrium is characterized by a system of inclusions, not by that of equations. As the second and central achievement, we show the robust indeterminacy of the stationary monetary equilibrium, which is a time-invariant equilibrium with circulating money. To make the meaning of robustness clear, let us call a pair of initial endowments in the first and second periods of the agent's life an economy. Then the second result is that there is a nonempty open set of economies, each element of which generates a continuum of stationary monetary equilibria.

libria in an intertemporal general equilibrium model with an infinitely-lived representative agent. Note, however, that they only demonstrated nominal indeterminacy in this setting; i.e., they demonstrated the indeterminacy of the equilibrium asset prices but not of the equilibrium allocation. In their model, the single representative agent consumes the sum of the initial endowment and the dividend at each date along the equilibrium price path, and hence, real indeterminacy does not occur by the definition of the equilibrium. 
Furthermore, we argue that such economies exhibit aggregate uncertainty. Thirdly, we conduct comparative statics of the ambiguity. We show that an increase in ambiguity (in the sense that the set of priors dilates) increases the sizes of both the range of the stationary monetary equilibria and the set of economies generating a continuum of stationary monetary equilibria. Finally, we show that each of these stationary monetary equilibria is conditionally Pareto optimal. In other words, the equilibria are not Pareto comparable with each other.

The paper is organized as follows. Section 2 presents some relevant results from the existing literature. Section 3 describes the details of our model. Section 4 provides two-state examples that illustrate the mechanism generating indeterminacy of equilibria. Section 5 demonstrates that stationary monetary equilibrium can be characterized by a system of inclusions and then shows the existence and robust indeterminacy of stationary monetary equilibria. Section 6 conducts comparative statics that illustrates the effect of greater ambiguity on the equilibrium outcomes. Section 7 examines the optimality of stationary monetary equilibria. Proofs are collected in the final section.

\section{Related Literature}

This paper builds on a number of contributions. As mentioned in the Introduction, the static Arrow-Debreu model under ambiguity can generate indeterminacy of equilibria (for example, when there exists no aggregate uncertainty). However, such indeterminacy is not robust. To generate robust indeterminacy in a general equilibrium setting under ambiguity, "some other ingredient has to be inserted" as proposed by Mukerji and Tallon (2004, P.285). The most recent studies found some situations that exhibit robust indeterminacy in a general equilibrium setting under ambiguity. Mukerji and Tallon (2001, Theorem) incorporated an idiosyncratic component of the asset returns into the Arrow-Debreu model to argue the incompleteness of financial markets and indeterminacy of equilibria. ${ }^{6}$ Furthermore, Mandler (2013, Proposition 1)

\footnotetext{
${ }^{6}$ See Rinaldi (2009) for additional information. Note, however, that since the MEU preference is a special case of the variational preference axiomatized by Maccheroni, Marinacci and Rustihici (2006) and since any variational preference may be approximated arbitrarily well by another smooth variational preference, Rinaldi's (2009) result is not a confirmation that Mukerji and Tallon's (2001) result works for the whole class of variational preferences. In a similar fashion, the robust indeterminacy in this paper is concerned with the robustness with respect to only the initial endowments and not the robustness with respect to both the initial endowments and the preferences. That is, the preferences are supposed to be fixed. The authors are grateful to an anonymous referee for making this point clear.
} 
recently developed a near Arrow-Debreu model by introducing a productive asset and sequential trades. He found robust indeterminacy of equilibria. In his model, the production was used to drive the economy to a point where agents face decision-making in a setting in which there exist no aggregate uncertainty. The observation made in this paper contributes to this line of the literature by providing a new mechanism which generates robust indeterminacy of equilibria in a general equilibrium model under ambiguity. That is, we use the intuition obtained from the static Arrow-Debreu model and embed ambiguity into a stochastic OLG model with money. We can adjust the value of money in each state to make the environment similar to the one with no aggregate uncertainty. One may consider that this mechanism is, in some sense, similar to Mandler's (2013). However, they are quite distinct from each other because, in our model, the flexible adjustment of the real price of money (not the production) makes the environment with which the agents is faced similar to the static one. In a summary, we could say that, by introducing a demographic structure with overlapping generations and an infinitely-lived asset (money) in a constant supply to an intertemporal general equilibrium setting, we can generate robust indeterminacy of equilibria under ambiguity.

Our observations also contribute to studies on stochastic OLG models of the general equilibrium. The proposed model is simple but rather canonical and similar to those considered in the literature. For examples, see Magill and Quinzii (2003), Labadie (2004) and Ohtaki (2011). However, the central subjects of these preceding works are different from ours. ${ }^{7}$ We should note that this paper is the first that introduces the MEU preferences to the stochastic OLG model with money. ${ }^{8}$

Our model is canonical except for the preferences, but the density of the stationary monetary equilibria is quite different from the preceding studies. In representative agent models, Manuelli (1990, Proposition 1), Magill and Quinzii (2003, Proposition 1) and Ohtaki (2011, Proposition $1^{\prime}$ ) reported several sufficient conditions for the uniqueness of stationary monetary equilibrium. ${ }^{9}$ Furthermore, Gottardi (1996, Theorem 1) showed that a stationary monetary equi-

\footnotetext{
${ }^{7}$ Labadie (2004) explored financial arrangements that could realize the optimal allocations. Magill and Quinzii (2003) examined the asymptotic properties of monetary equilibrium processes.

${ }^{8}$ Fukuda (2008) introduced firms with convex Choquet expected utility preferences to Diamond's (1965) OLG model with a capital accumulation.

${ }^{9}$ Interested readers may also see Ohtaki (2013b).
} 
librium generically exists and is locally isolated in a more complicated stochastic OLG model with many one-period securities and many agents endowed with smooth preferences. With the MEU preferences which is known to be nonsmooth, in contrast, we show that the indeterminacy of stationary monetary equilibria can arise even when the sufficient conditions provided in the preceding works for the uniqueness of stationary monetary equilibrium are satisfied.

Finally, we mention our observation on the optimality of equilibria. Optimality of equilibrium allocations has been characterized by Manuelli (1990), Aiyagari and Peled (1991), Chattopadhyay and Gottardi (1999), Demang and Laroque (1999) and Ohtaki (2013a) among others. These studies presumed that the preferences were smooth. However, the MEU preferences are nonsmooth as mentioned in the previous paragraph. Therefore, we cannot directly apply the characterization results developed in these preceding studies to the current model. ${ }^{10}$ Instead of applying the established characterization results, we have more directly demonstrated the optimality of stationary monetary equilibria by tailoring Sakai's (1988) proof to our model.

\section{The Model}

We consider a stationary, pure-endowment, stochastic overlapping generations economy with money, where agents' beliefs about uncertain situations are represented by multiple priors, not by a single prior. ${ }^{11}$

\subsection{Stochastic Environment}

Time is discrete and runs from $t=1$ to infinity. Uncertainty is modeled by a date-event tree with a time-invariant finite state space $S$. For each $t \geq 1$, we denote the state realized in period $t$ as $s_{t}$. This is called the period $t$ state, where the (conceptually defined) period 0 state $s_{0} \in S$ is treated as given. To be more precise, the date-event tree, denoted by $\Gamma$, is defined as follows. (i) The root of the tree is $s_{0}$; (ii) the set of nodes at date $t$ is denoted by $\Sigma_{t}$ (where we set $\Sigma_{1}:=\left\{s_{0}\right\} \times S$ and $\Sigma_{t}:=\Sigma_{t-1} \times S$ for $t \geq 2$ ); and (iii) $\Sigma:=\bigcup_{t \geq 1} \Sigma_{t}$ and $\Gamma:=\left\{s_{0}\right\} \cup \Sigma .^{12}$ In

\footnotetext{
${ }^{10}$ Recently, Ohtaki and Ozaki (2013) extended the standard dominant root characterization of the optimal allocations to the economy under ambiguity.

${ }^{11}$ Except for the preferences, the ingredients of our model are similar to those in Labadie's work (2004). However, her objective was to examine the financial arrangements that could result in the optimal allocations, not to examine the in/determinacy of stationary monetary equilibrium.

${ }^{12}$ This definition of the date-event tree is standard, and can be seen in, for example, Chattopadhyay (2001).
} 
each period, we assume that the state is realized at the beginning of the period. We denote the set of all probability measures on $S$ as $\Delta_{S}$. There is a single perishable commodity, called the consumption good in each period. No storage technology nor production technology is available.

In each period, one new agent enters the economy after the state is realized and lives for two periods. In the rest of this paper, we concentrate on the stationary situation. Therefore, agents are distinguished by the state in which they are born, and not the time nor the past history of the realized states. An agent born in state $s_{t}$ and period $t$ is endowed with $\omega_{s t}^{y}$ units of the consumption good in the first period of their life, and $\omega_{s_{t+1}}^{o}$ in state $s_{t+1}$ in the second period. We assume that $\left(\omega_{s}^{y},\left(\omega_{s^{\prime}}^{o}\right)_{s^{\prime} \in S}\right) \in \Re_{++} \times \Re_{++}^{S}$ for all $s \in S$. Note that the secondperiod endowment is assumed to be independent of the shock in the first period. Therefore, the economy is represented by a point $\left(\omega_{s}^{y}, \omega_{s^{\prime}}^{o}\right)_{s, s^{\prime} \in S}$ in the positive orthant of the finite-dimensional Euclidean space, $\Re_{++}^{S \times S}$, given the agent's preferences described below.

\subsection{Preferences}

We denote the contingent consumption stream of an agent born in state $s_{t}$ at date $t$ as $c_{s_{t}}=\left(c_{s_{t}}^{y},\left(c_{s_{t} s^{\prime}}^{o}\right)_{s^{\prime} \in S}\right) \in \Re_{+} \times \Re_{+}^{S}$. Each agent born in state $s_{t}$ at date $t$ is assumed to rank the consumption streams $c_{s_{t}}$ according to their lifetime utility function $U^{s_{t}}: \Re_{+} \times \Re_{+}^{S} \rightarrow \Re$. Furthermore, each agent born in state $s$ is assumed to form a belief about an occurrence of the next period's state that is represented by a set of priors which are totally subjective, given the state realized in the period when they were born. ${ }^{13}$ Throughout this paper, we assume that an agent born in state $s$ has an MEU preference. ${ }^{14}$ That is, there exists a strictly monotone increasing and strictly concave real-valued function $u$ on $\Re_{+} \times \Re_{+}$, which is continuously differentiable on the interior of its domain, and a compact and convex subset $\mathcal{P}_{s}$ of $\Delta_{S} \cap \Re_{++}^{S}$ such that

$$
\left(\forall c_{s} \in \Re_{+} \times \Re_{+}^{S}\right) \quad U^{s}\left(c_{s}\right)=\min _{\pi_{s} \in \mathcal{P}_{s}} \sum_{s^{\prime} \in S} u\left(c_{s}^{y}, c_{s s^{\prime}}^{o}\right) \pi_{s s^{\prime}} .
$$

Note that we assume that $u$ is common to all agents. When $\mathcal{P}_{s}$ is a singleton for each $s \in S$, the preference degenerates into the standard subjective expected utility. In such a case, $\mathcal{P}_{s}=\left\{\pi_{s}\right\}$

\footnotetext{
${ }^{13}$ That is, the formation of the belief is independent of the past history of realized states. Further, the set of priors may not be common to all agents who are distinguished by the states at which they are born.

${ }^{14}$ Gilboa and Schmeidler (1989) axiomatized the MEU preferences over lottery acts and Casadesus-Masanell, Klibanoff and Ozdenoren (2000) axiomatized the MEU preferences over Savage acts. Their axiomatization does not depend on whether the state space is finite or infinite, and hence, it may be applied to our situation with a finite state space.
} 
for some $\pi_{s} \in \Delta_{S}$ for each $s$ and we may write $U^{s}\left(c_{s}\right)$ as $U\left(\pi_{s}\right)(c)$ since $U^{s}$ does not depend on $s$ directly by the commonality of $u$. Then, because $U\left(\pi_{s}\right)\left(c_{s}\right)$ is clearly continuous in $\pi_{s}$ for each $c_{s}$ and because $\mathcal{P}_{s}$ is compact by the assumption, the minimum in (1) can be actually achieved. And hence, $U^{s}$ is well-defined. We often write $U^{s}\left(c_{s}\right)$ as $U_{\mathcal{P}_{s}}\left(c_{s}\right)$ again because $U^{s}$ depends on $\mathcal{P}_{s}$, not directly on $s$. We define the nonempty set of priors which achieve the minimum in (1) by $\mathcal{M}$ as follows:

$$
(\forall s \in S)\left(\forall c_{s} \in \Re_{+} \times \Re_{+}^{S}\right) \quad \mathcal{M}_{s}\left(c_{s}\right):=\underset{\pi_{s} \in \mathcal{P}_{s}}{\arg \min } U\left(\pi_{s}\right)\left(c_{s}\right) .
$$

One can easily verify that this lifetime utility function is strictly concave because of the strict concavity of $u$. We will use this fact when we characterize the equilibria and when we show their conditional Pareto optimality.

Remark 1 The present model is very close to those of Magill and Quinzii (2003) and Labadie (2004). ${ }^{15}$ However, the interpretations on the agents' beliefs in this paper and in previous works are different. For example, Magill and Quinzii (2003) require that the (unique) subjective probability measure should coincide with the true probability measure that follows from a Markov process. On the other hand, in the present model, subjective beliefs does not necessarily coincide with the true or objective probability measures even when such measures exist. In other words, the formation of the agent's belief which is represented by a set of priors, whether it is a singleton or not, is totally subjective and it could be totally irrelevant to the true or objective probability measures. This interpretation on the agents' beliefs might be considered as a kind of bounded rationality and is different from those of Magill and Quinzii (2003) and Labadie (2004).

We sometimes write $\mathcal{P}=\left(\mathcal{P}_{s}\right)_{s \in S}$ and call it the transition probability correspondence. Here, we give an example of the transition probability correspondence.

Example 1 Suppose that there exist some $\hat{\pi}: S \rightarrow \Delta_{S}$ and $\varepsilon: S \rightarrow[0,1]$ such that, for each $s \in S, \mathcal{P}_{s}=\left\{\left(1-\varepsilon_{s}\right) \hat{\pi}_{s}+\varepsilon_{s} \mu_{s}: \mu_{s} \in \Delta_{S}\right\}$, which is often called the $\varepsilon$-contamination of $\hat{\pi}_{s}{ }^{16}$ Obviously, this is an example of transition probability correspondences. Then, the associated

\footnotetext{
${ }^{15}$ Labadie (2004) considered a time-separable utility index function.

${ }^{16}$ The $\varepsilon$-contamination was first axiomatized by Nishimura and Ozaki (2006).
} 
lifetime utility function (1) takes the form:

$$
U^{s}\left(c_{s}\right)=\min _{\pi_{s} \in \mathcal{P}_{s}} \sum_{s^{\prime} \in S} u\left(c_{s}^{y}, c_{s s^{\prime}}^{o}\right) \pi_{s s^{\prime}}=\left(1-\varepsilon_{s}\right) \sum_{s^{\prime} \in S} u\left(c_{s}^{y}, c_{s s^{\prime}}^{o}\right) \hat{\pi}_{s s^{\prime}}+\varepsilon_{s} \min _{s^{\prime} \in S} u\left(c_{s}^{y}, c_{s s^{\prime}}^{o}\right) .
$$

Note that an increase in $\varepsilon_{s}$ increases the ambiguity in the sense that $\mathcal{P}_{s}$ dilates. The effect of this type of increase in ambiguity will be studied in Section 7 .

\subsection{Stationary Monetary Equilibrium}

We next turn to the definition of a stationary monetary equilibrium, a stationary equilibrium wherein money circulates. To describe the intergenerational trade via money, we introduce an infinitely-lived outside asset that yields no dividends and is in constant supply. We denote the stock of fiat money as $M>0$, which is constant over the date-events. We also let $p_{s}$ be the real price of the money and $q_{s}$ be the real money balance at state $s \in S$. By definition, $p_{s} M=q_{s}$ and therefore $p_{s}$ and $q_{s}$ has a one-to-one correspondence. A stationary monetary equilibrium is then defined by a pair $\left(q^{*}, c^{*}\right)$ of a contingent real money balance $q^{*} \in \Re_{++}^{S}$ and a contingent consumption stream $c^{*}=\left(c_{s}^{* y},\left(c_{s s^{\prime}}^{* o}\right)_{s^{\prime} \in S}\right)_{s \in S}$, such that there exists an $m^{*} \in \Re^{S}$ satisfying that, for all $s \in S$, (i) $\left(c_{s}^{*}, m_{s}^{*}\right)$ belongs to the set

$$
\underset{\left(c_{s}^{y},\left(c_{s s^{\prime}}^{o}\right)_{s^{\prime} \in S}, m_{s}\right) \in \Re_{++} \times \Re_{++}^{S} \times \Re}{\arg \max }\left\{U^{s}\left(c_{s}\right) \mid \begin{array}{c}
c_{s}^{y}=\omega_{s}^{y}-p_{s}^{*} m_{s}, \\
\left(\forall s^{\prime} \in S\right) c_{s s^{\prime}}^{o}=\omega_{s^{\prime}}^{o}+p_{s^{\prime}}^{*} m_{s}
\end{array}\right\}
$$

given $p_{\tau}^{*}:=q_{\tau}^{*} / M$ for each $\tau \in S$ and (ii) $m_{s}^{*}=M$.

Condition (i) requires that the pair of the consumption stream $\left(c_{s}^{* y},\left(c_{s s^{\prime}}^{* o}\right)_{s^{\prime} \in S}\right)$ and money holding $m_{s}^{*}$ must be the solution of the (lifetime) utility-maximizing problem of the agent born in state $s$. Condition (ii) is the market-clearing condition of fiat money. ${ }^{17}$ We can easily verify that, in any stationary monetary equilibrium, the market-clearing conditions for the contingent commodities hold (the Walras' law). That is, for each $s, s^{\prime} \in S, c_{s}^{* y}+c_{s^{\prime} s}^{* o}=\omega_{s}^{y}+\omega_{s}^{o}$. This also implies that the second-period consumptions at a stationary monetary equilibrium are independent of the state realized in the first-period of an agent's life; i.e., $c_{s^{\prime} s}^{* o}=c_{s^{\prime \prime} s}^{* o}$ for each $s, s^{\prime}, s^{\prime \prime} \in S$.

\footnotetext{
${ }^{17}$ In (i), we assume that the budget constraints hold with equalities. We can do this for the first budget constraint without loss of generality by the strict increase of $u$. For the other budget constraints, we simply assume it. Also note that we exclude corner solutions by assuming that $\left(c^{y},\left(c_{s^{\prime}}^{o}\right)_{s^{\prime} \in S}\right) \in \Re_{++} \times \Re_{++}^{S}$ and (ii).
} 
A stationary monetary equilibrium is called fully-insured (with respect to the second-period consumptions) if, for each $s, s^{\prime}, s^{\prime \prime} \in S, c_{s s^{\prime}}^{* o}=c_{s s^{\prime \prime}}^{* o}$. This type of stationary monetary equilibrium plays an important role in the following analyses.

Finally, note that, after an equilibrium real money balance $q^{*}$ has been chosen (and the money market has cleared), the equilibrium consumption stream $c^{*}$ is automatically and uniquely determined from the budget constraints. Therefore, we can identify a stationary monetary equilibrium $\left(q^{*}, c^{*}\right)$ with an equilibrium positive real money balance $q^{*}$. By using this convenient property, the agents' optimization problems can be simplified to the ones of choosing an optimal money holding. For later references, we define the utility function $V_{s}$ of the money holding $m_{s}$ for each agent born at state $s$ facing the real money balance $q^{*} \in \Re_{++}^{S}$. Formally, for each $s \in S$, let

$$
\left(\forall q^{*}\right)\left(\forall m_{s}\right) \quad V_{s}^{q^{*}}\left(m_{s}\right):=U_{\mathcal{P}_{s}}\left(c_{s}^{m_{s}}\left(q^{*}\right)\right)
$$

where $c_{s s^{\prime}}^{m_{s}}\left(q^{*}\right):=\left(\omega_{s}^{y}-\left(q_{s}^{*} / M\right) m_{s}, \omega_{s^{\prime}}^{o}+\left(q_{s^{\prime}}^{*} / M\right) m_{s}\right)$ and $c_{s}^{m_{s}}\left(q^{*}\right):=\left(\omega_{s}^{y}-\left(q_{s}^{*} / M\right) m_{s},\left(\omega_{s^{\prime}}^{o}+\right.\right.$ $\left.\left.\left(q_{s^{\prime}}^{*} / M\right) m_{s}\right)_{s^{\prime} \in S}\right)$. Note that $V_{s}$ is concave because of the strict concavity of $u$. We often suppress the superscript $q^{*}$ when it is obvious from the context.

\section{Two-state Illustrative Examples}

Before conducting formal analyses, this section offers two examples which provide intuitions for the mechanism which generates indeterminacy of equilibria.

The next example assumes only two states and constructs economies which exhibit indeterminacy of equilibria. In particular, it shows that the indeterminacy is caused by the nondifferentiability of the MEU preferences at consumption streams which are fully-insured with respect to the second-period consumptions.

Example 2 Suppose that $S=\{\alpha, \beta\}$. Additionally, specify the model by assuming that $\left(\omega_{\alpha}^{y}, \omega_{\beta}^{y}, \omega_{\alpha}^{o}, \omega_{\beta}^{o}\right)=(6,3,1,2), u\left(c_{s}^{y}, c_{s s^{\prime}}^{o}\right)=\ln c_{s}^{y}+\ln c_{s s^{\prime}}^{o}$, and $\mathcal{P}_{s}=\left\{\left(\pi_{s \alpha}, \pi_{s \beta}\right) \in \Delta_{S} \mid \varepsilon \leq \pi_{s \alpha} \leq \delta\right\}$ for each $s \in S$, where $0<\varepsilon \leq \delta<1 .^{18}$ Because we consider a two-state model, the optimization

${ }^{18}$ The MEU preferences in the Cobb-Douglas form as in this example have recently been axiomatized by Faro (2013). 


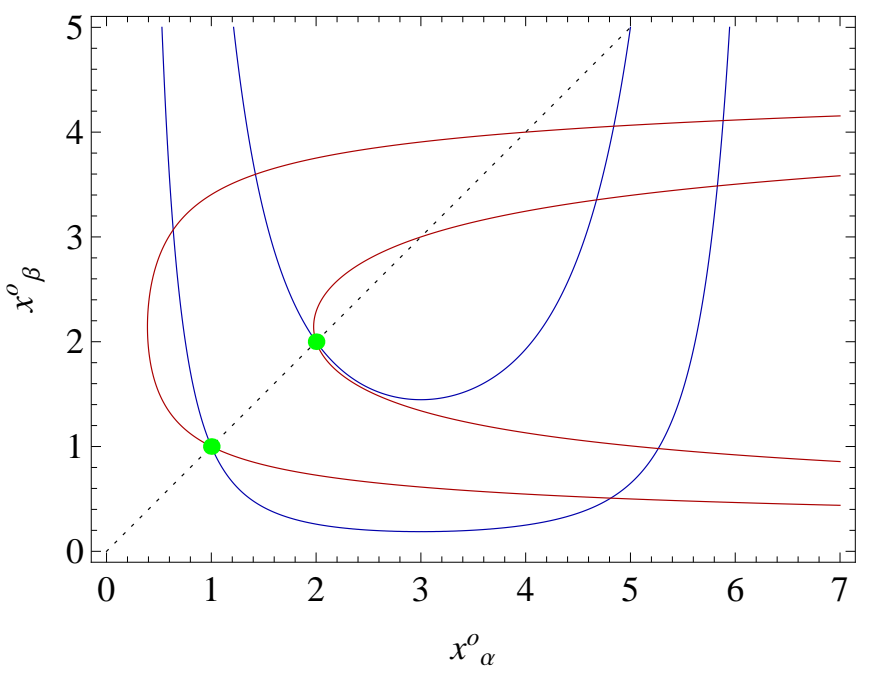

a. $(\varepsilon, \delta)=(0.5,0.5)$

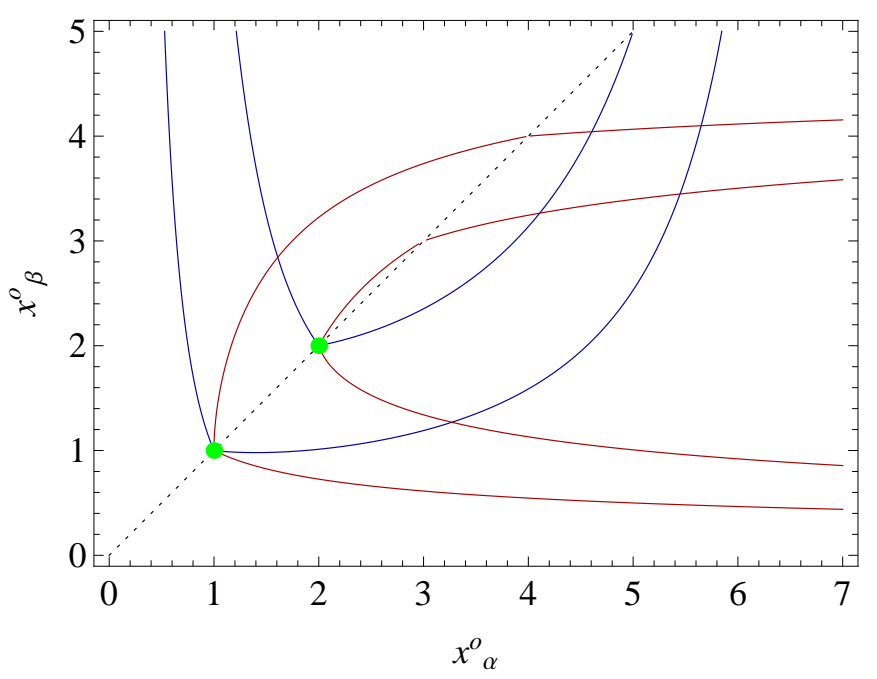

b. $(\varepsilon, \delta)=(0.25,0.75)$

Figure 1: "Edgeworth box": (a) there is no ambiguity, (b) there is ambiguity.

problem in Condition (i) of the definition of stationary monetary equilibrium can be rewritten as

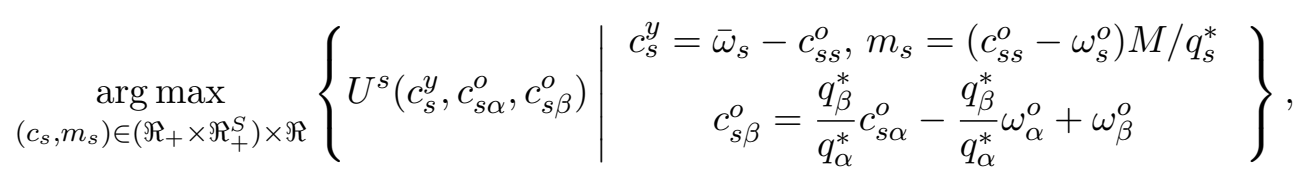

where $\bar{\omega}_{s}:=\omega_{s}^{y}+\omega_{s}^{o}$. In this condition, $c_{s}^{y}$ and $m_{s}$ completely depend on the selection of $\left(c_{s \alpha}^{o}, c_{s \beta}^{o}\right)$, especially $c_{s s}^{o}$, for each $s \in S$. Furthermore, the first constraint, $c_{s}^{y}=\bar{\omega}_{s}-c_{s s}^{o}$, implies that $\left(c_{s \alpha}^{o}, c_{s \beta}^{o}\right) \in X_{s}:=\left\{\left(x_{\alpha}^{o}, x_{\beta}^{o}\right) \in \Re_{+}^{2} \mid x_{s}^{o} \leq \bar{\omega}_{s}\right\}$ because $c_{s}^{y}, c_{s s}^{o} \geq 0$. Therefore, we can consider that each agent born at state $s \in S$ chooses $\left(x_{\alpha}^{o}, x_{\beta}^{o}\right) \in X_{s}$ so as to maximize $\hat{U}^{s}\left(x_{\alpha}^{o}, x_{\beta}^{o}\right):=U^{s}\left(\bar{\omega}_{s}-x_{s}^{o}, x_{\alpha}^{o}, x_{\beta}^{o}\right)$ subject to the "budget line", $x_{\beta}^{o}=\left(q_{\beta}^{*} / q_{\alpha}^{*}\right) x_{\alpha}^{o}-\left(q_{\beta}^{*} / q_{\alpha}^{*}\right) \omega_{\alpha}^{o}+\omega_{\beta}^{o}$, between two commodities contingent upon state $\alpha$ and $\beta$. This means that we can consider solutions of the MEU-maximizing problem and stationary monetary equilibrium using a "box diagram" with indifference curves derived from $\hat{U}^{s}$ and the budget line. In Figure 1, the boxes depict the space $X_{\alpha} \cap X_{\beta}$ and indifference curves, derived from $\hat{U}^{s}$, through $\left(x_{\alpha}, x_{\beta}\right)=(1,1)$ and $\left(x_{\alpha}, x_{\beta}\right)=(2,2)$. The $U$-shaped and $\subset$-shaped curves are related to indifference curves for agents born at states $\alpha$ and $\beta$, respectively. Note that $\hat{U}^{s}(1,1)<\hat{U}^{s}(2,2)$. It is assumed that $\varepsilon=\delta=0.5$ in Figure $1(\mathrm{a})$ and $(\varepsilon, \delta)=(0.25,0.75)$ in Figure 1(b). Therefore, Figures 1(a) and 1(b) are related to cases without and with ambiguity, respectively. Notably, the agent's indifference curves in Figure 1(b) exhibit kinks at the 45-degree line. This is because the lifetime 


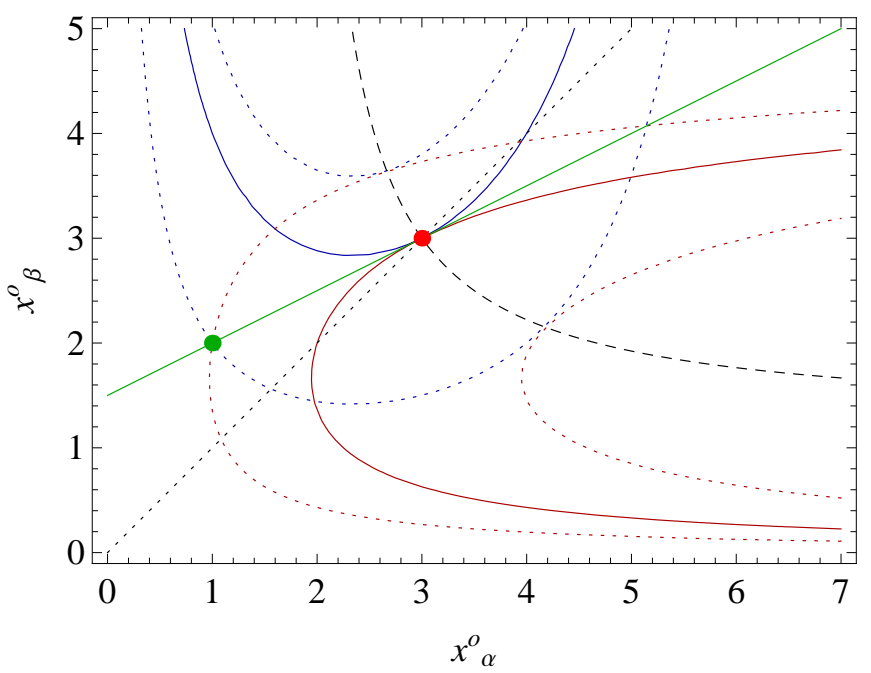

a. $(\varepsilon, \delta)=(0.5,0.5)$

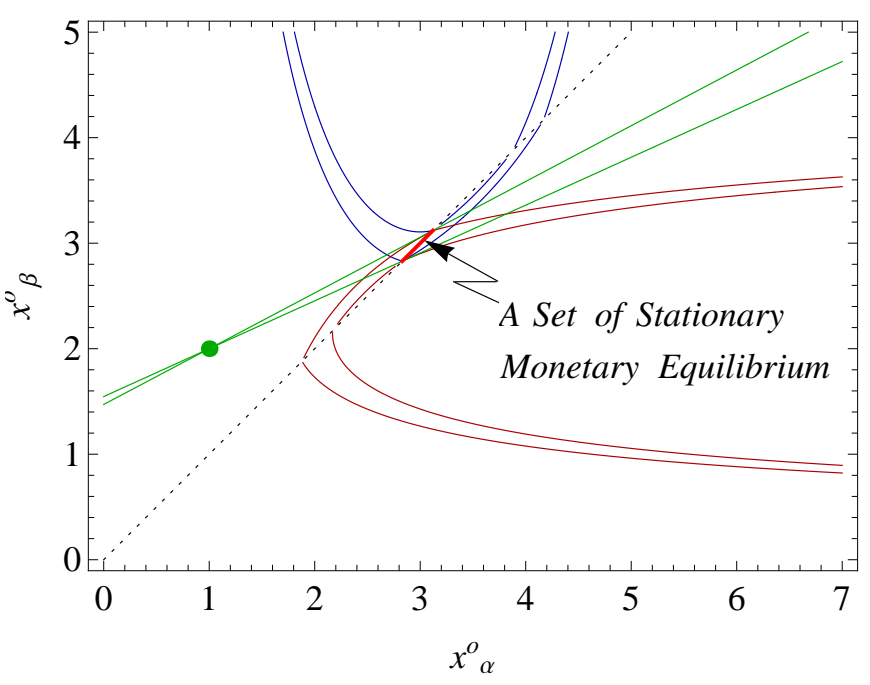

b. $(\varepsilon, \delta)=(0.25,0.75)$

Figure 2: Stationary monetary equilibria: (a) there is no ambiguity; (b) there is ambiguity.

MEU function is not differentiable on this line. ${ }^{19}$ Figure 2 introduces the budget lines to the box diagram and depicts the stationary monetary equilibria. In this figure, the point $\left(x_{\alpha}^{o}, x_{\beta}^{o}\right)=(1,2)$ corresponds to the initial endowment, and straight lines through it represent the "budget lines". A second-period consumption $\left(x_{\alpha}^{o}, x_{\beta}^{o}\right)$ at a stationary monetary equilibrium then corresponds to a point on a "budget line" when it is tangential to the indifference curves of both agents $(\alpha$ and $\beta$ ). When $\varepsilon=\delta$ (i.e., there exists no ambiguity), we can find a unique stationary monetary equilibrium, as in Figure 2(a). Here, the dashed line depicts the set of points at which the indifference curves of agents $\alpha$ and $\beta$ are tangential to each other. ${ }^{20}$

When $\varepsilon<\delta$ (i.e., there exists ambiguity), there exists a continuum of stationary monetary

\footnotetext{
${ }^{19}$ To be more precise, the marginal rates of substitution for indifferent curves derived from $\hat{U}^{s}$, denoted by $\widehat{M R S}_{s}\left(x^{\circ}\right)$, is calculated using

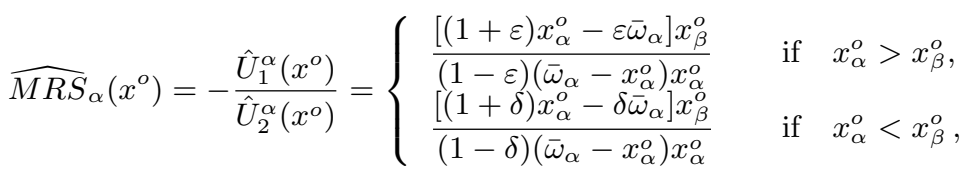

and

$$
\widehat{M R S}_{\beta}\left(x^{o}\right)=-\frac{\hat{U}_{1}^{\beta}\left(x^{o}\right)}{\hat{U}_{2}^{\beta}\left(x^{o}\right)}= \begin{cases}\frac{\varepsilon\left(\bar{\omega}_{\beta}-x_{\beta}^{o}\right) x_{\beta}^{o}}{\left[(2-\varepsilon) x_{\alpha}^{o}-(1-\varepsilon) \bar{\omega}_{\beta}\right] x_{\alpha}^{o}} & \text { if } \quad x_{\alpha}^{o}>x_{\beta}^{o}, \\ \frac{\delta\left(\bar{\omega}_{\beta}-x_{\beta}^{o}\right) x_{\beta}^{o}}{\left[(2-\delta) x_{\alpha}^{o}-(1-\delta) \bar{\omega}_{\beta}\right] x_{\alpha}^{o}} & \text { if } \quad x_{\alpha}^{o}<x_{\beta}^{o}\end{cases}
$$

if $x_{\alpha}^{o} \neq x_{\beta}^{o}$. However, it cannot be calculated at $x^{o}$ with $x_{\alpha}^{o}=x_{\beta}^{o}$, if $\varepsilon<\delta$.

${ }^{20}$ To be more precise, the uniqueness of stationary monetary equilibrium follows from, for example, Proposition 1 of Magill and Quinzii (2003) or Theorem 1 of Ohtaki (2013b).
} 
equilibria, as in Figure 2(b). Here, a second-period consumption $x^{o}=x_{\alpha}^{o}=x_{\beta}^{o}$ that corresponds to some stationary monetary equilibrium lies on the open interval of approximately $2.83-$ $-3.12 .^{21}$

The previous example suggests that the indeterminacy of equilibria arises because of nonsmoothness of the agents' MEU preferences and how to characterize such equilibria is not yet clear. The next example also assumes only two states and gives an intuition for the characterization of the stationary monetary equilibria under ambiguity.

Example 3 Suppose that $S=\{\alpha, \beta\}$ and $\omega_{\alpha}^{o}<\omega_{\beta}^{o}$. Because $\mathcal{P}_{s}$ is compact and convex for each $s \in S$, we can write it as $\mathcal{P}_{s}=\left\{\pi_{s} \in \Delta_{S} \mid \mu_{s \alpha} \leq \pi_{s \alpha} \leq \nu_{s \alpha}\right\}$ for some $\mu_{s}, \nu_{s} \in \Delta_{S}$ such that $0<\mu_{s \alpha}<\nu_{s \alpha}<1$. Additionally, suppose that $u\left(c_{s}^{y}, c_{s s^{\prime}}^{o}\right)=v_{y}\left(c_{s}^{y}\right)+v_{o}\left(c_{s s^{\prime}}^{o}\right)$ for some strictly monotone increasing and strictly concave real-valued functions $v_{y}$ and $v_{o}$ on $\Re_{+}$, which are continuously differentiable on the interior of its domain. Assume that there exists a stationary monetary equilibrium $\left(q^{*}, c^{*}\right)$, wherein $\omega_{\alpha}^{o}+q_{\alpha}^{*}=\omega_{\beta}^{o}+q_{\beta}^{*}:=\bar{c}^{* o}$ for each $s, s^{\prime} \in S$. We have observed in Example 2 that there exists at least one economy generating such an equilibrium. Note that $q_{\alpha}^{*}>q_{\beta}^{*}$ because $\omega_{\alpha}^{o}<\omega_{\beta}^{o}$, and $\sum_{s^{\prime} \in S} q_{s^{\prime}}^{*} \mu_{s s^{\prime}}<\sum_{s^{\prime} \in S} q_{s^{\prime}}^{*} \nu_{s s^{\prime}}$ for each $s \in S$. Then, we can show that $V_{s}$ is not differentiable at $M$, which must be chosen at any stationary monetary equilibrium. This is easy to verify. First, we can obtain

$$
V_{s}(M)=v_{y}\left(\omega_{s}^{y}-q_{s}^{*}\right)+v_{o}\left(\bar{c}^{* o}\right)
$$

and, for any real number $h$ that is sufficiently close to but not equal to 0 ,

$$
\begin{aligned}
V_{s}(M+h) & =v_{y}\left(\omega_{s}^{y}-q_{s}^{*}-\left(q_{s}^{*} / M\right) h\right)+\min _{\pi_{s} \in \mathcal{P}_{s}} \sum_{s^{\prime} \in S} v_{o}\left(\bar{c}^{* o}+\left(q_{s^{\prime}}^{*} / M\right) h\right) \pi_{s s^{\prime}} \\
& =v_{y}\left(\omega_{s}^{y}-q_{s}^{*}-\left(q_{s}^{*} / M\right) h\right)+ \begin{cases}\sum_{s^{\prime} \in S} v_{o}\left(\bar{c}^{* o}+\left(q_{s^{\prime}}^{*} / M\right) h\right) \mu_{s s^{\prime}} & \text { if } \quad h>0, \\
\sum_{s^{\prime} \in S} v_{o}\left(\bar{c}^{* o}+\left(q_{s^{\prime}}^{*} / M\right) h\right) \nu_{s s^{\prime}} & \text { if } \quad h<0\end{cases}
\end{aligned}
$$

because $q_{\alpha}^{*}>q_{\beta}^{*}$. Then, it follows that

$$
R_{s}:=\lim _{h \downarrow 0} \frac{V_{s}(M+h)-V_{s}(M)}{h}=-\frac{q_{s}^{*}}{M} v_{y}^{\prime}\left(\omega_{s}^{y}-q_{s}^{*}\right)+\frac{1}{M} v_{o}^{\prime}\left(\bar{c}^{* o}\right) \sum_{s^{\prime} \in S} q_{s^{\prime}}^{*} \mu_{s s^{\prime}}
$$

\footnotetext{
${ }^{21}$ The detail of the box diagram presented here was provided in Ohtaki (2014).
} 
and

$$
L_{s}:=\lim _{h \uparrow 0} \frac{V_{s}(M+h)-V_{s}(M)}{h}=-\frac{q_{s}^{*}}{M} v_{y}^{\prime}\left(\omega_{s}^{y}-q_{s}^{*}\right)+\frac{1}{M} v_{o}^{\prime}\left(\bar{c}^{* o}\right) \sum_{s^{\prime} \in S} q_{s^{\prime}}^{*} \nu_{s s^{\prime}} .
$$

Therefore, $V_{s}$ is not differentiable at $M$ because $R_{s}<L_{s}$. However, we should remark that $M$ maximizes $V_{s}$ if and only if $R_{s} \leq 0 \leq L_{s}$; i.e., given $s \in S$

$$
\sum_{s^{\prime} \in S} \frac{q_{s^{\prime}}^{*}}{q_{s}^{*}} \mu_{s s^{\prime}} \leq \frac{v_{y}^{\prime}\left(c_{s}^{* y}\right)}{v_{o}^{\prime}\left(\bar{c}^{* o}\right)} \leq \sum_{s^{\prime} \in S} \frac{q_{s^{\prime}}^{*}}{q_{s}^{*}} \nu_{s s^{\prime}}
$$

which gives us a partial characterization of the stationary monetary equilibrium.

\section{Robust Indeterminacy}

This section presents the first two out of four achievements we mentioned in the Introduction and constitutes the main body of this paper. Subsection 5.1 characterizes the stationary monetary equilibria by the solutions to the system of difference inclusions. Subsection 5.2 shows that there exists a continuum of equilibria and that it is robust in the sense that there exists an open set of economies all elements of which generate such indeterminacy of equilibria.

\subsection{Characterization of Stationary Monetary Equilibria}

The inequalities in (3) in Example 3 can be interpreted as a requirement that, in a stationary monetary equilibrium, the marginal rate of substitution between the first- and second-period consumptions lies in the range of expected rates of the return of the money. We may thus say that (3) represents a trade-off existing between intertemporal consumption smoothing and aversion to ambiguity and that agents can avoid ambiguity in the second-period consumption by sacrificing smoothing between the first- and second-period consumptions. Furthermore, the range of the marginal rates of substitution which are admitted at a solution of the agents' optimization problems is nondegenerate by the presence of ambiguity. All of these suggest that, under the MEU preference, a stationary monetary equilibrium may be characterized by the solution to the system of difference inclusions, not of difference equations.

The conjecture made in the previous paragraph is in fact correct as we show below. It is well-known that if a stationary monetary equilibrium exists, it can be characterized by the solution to the system of difference equations that are derived from the first-order conditions of 
the agents' optimization problems provided the agents' preferences are represented by standard smooth utility functions. ${ }^{22}$ In contrast, the stationary monetary equilibria in our model with the MEU preference, if they exist, can be characterized by the solutions to the system of difference inclusions.

Theorem 1 A positive vector $q^{*} \in \Re_{++}^{S}$ is a stationary monetary equilibrium if and only if

$$
(\forall s \in S) \quad 0 \in\left\{-\sum_{s^{\prime} \in S} q_{s}^{*} u_{1}\left(c_{s s^{\prime}}^{M}\left(q^{*}\right)\right) \pi_{s^{\prime}}+\sum_{s^{\prime} \in S} q_{s^{\prime}}^{*} u_{2}\left(c_{s s^{\prime}}^{M}\left(q^{*}\right)\right) \pi_{s^{\prime}} \mid \pi \in \mathcal{M}_{s}\left(c_{s}^{M}\left(q^{*}\right)\right)\right\},
$$

where $u_{i}$ is the derivative of $u$ with respect to the $i$-th argument.

This is a natural extension of the characterization of a stationary monetary equilibrium in a stochastic OLG model with a single prior. In fact, if $\mathcal{P}_{s}$ is a singleton for all $s \in S$, this system of inclusions degenerates into a system of equations. Then this result turns out to be the same as the one of a stochastic OLG model with a single prior, as shown in, for example, Definition 2 of Magill and Quinzii (2003).

To close this subsection, we provide the characterization of fully-insured stationary monetary equilibria, which is an immediate consequence of Theorem 1.

Corollary 1 A positive vector $q^{*} \in \Re_{++}^{S}$ such that $\omega_{s}^{o}+q_{s}^{*}=\omega_{s^{\prime}}^{o}+q_{s^{\prime}}^{*}=: \bar{c}^{* o}$ for each $s, s^{\prime} \in S$, is a fully-insured stationary monetary equilibrium if and only if

$$
(\forall s \in S) \quad \min _{\pi_{s} \in \mathcal{P}_{s}} \sum_{s^{\prime} \in S} \frac{q_{s^{\prime}}^{*}}{q_{s}^{*}} \pi_{s s^{\prime}} \leq \frac{u_{1}\left(c_{s}^{* y}, \bar{c}^{* o}\right)}{u_{2}\left(c_{s}^{* y}, \bar{c}^{* o}\right)} \leq \max _{\pi_{s} \in \mathcal{P}_{s}} \sum_{s^{\prime} \in S} \frac{q_{s^{\prime}}^{*}}{q_{s}^{*}} \pi_{s s^{\prime}},
$$

where $c_{s}^{* y}=\omega_{s}^{y}-q_{s}^{*}$.

The inequalities in (5) are obviously natural extensions of the inequalities in (3). Therefore, this result can also be interpreted as a trade-off between intertemporal consumption smoothing and aversion to ambiguity. We will exploit this corollary to find economies that certainly generate a continuum of stationary monetary equilibria.

\footnotetext{
${ }^{22}$ See, for example, Magill and Quinzii (2003) and Ohtaki (2011).
} 


\subsection{Existence and Robust Indeterminacy of Equilibria}

This subsection shows the robust indeterminacy of stationary monetary equilibria under the presence of ambiguity. That is, we demonstrate that there exists a nonempty open set of economies, each element of which generates a continuum of stationary monetary equilibria. To this end, we need to do two things. First, we need to show the existence of a continuum of stationary monetary equilibria. However, even when there exists no ambiguity, the stochastic OLG model with money may generate a continuum of nonstationary monetary equilibria (Magill and Quinzii, 2003, Proposition 2). ${ }^{23}$ On the other hand, a stationary monetary equilibrium can be unique when, for example, the preferences are time-separable and the degree of relative risk aversion of the second-period utility function is less than or equal to one. (Magill and Quinzii, 2003, Proposition 1). ${ }^{24}$ More generally, we can deduce from Gottardi (1996, Theorem 1) that the stationary monetary equilibrium is locally isolated and hence cannot be a continuum when the preferences are smooth. ${ }^{25}$ To present a stark contrast between stochastic OLG models with and without ambiguity, we demonstrate the indeterminacy of the stationary monetary equilibrium.

Second, we need to show that the indeterminacy is robust. We do this by showing the existence of a nonempty open set of economies in which the indeterminacy arises. This forces us to show that the indeterminacy may arise even when there exists the aggregate uncertainty. In fact, each element of the set of economies whose existence is to be proved exhibits the aggregate uncertainty (see Remark 3 below).

In the previous subsection, we observed that the stationary monetary equilibria can be characterized by the solutions to the system of difference inclusions. However, we have not yet shown the existence of such solutions. Consider an equilibrium real money balance $q^{*} \in \Re_{++}^{S}$, such that for any $s^{\prime}, s^{\prime \prime} \in S, \omega_{s^{\prime}}^{o}+q_{s^{\prime}}^{*} \neq \omega_{s^{\prime \prime}}^{o}+q_{s^{\prime \prime}}^{*}$ whenever $s^{\prime} \neq s^{\prime \prime}$. In this case, there exists a neighborhood of $q^{*}$ in which $\mathcal{M}_{s}\left(c_{s}^{M}(q)\right)$ is "constant and a singleton" for each $s .{ }^{26}$ We refer

\footnotetext{
${ }^{23}$ To be more precise, Magill and Quinzii (2003, Proposition 2) show the indeterminacy of the candidates for "expectation functions," each of which constructs a nonstationary rational expectations equilibrium with circulating money.

${ }^{24}$ See also Theorem of Ohtaki (2013b).

${ }^{25}$ As shown by Gottardi (1996), a "zero-th order" stationary monetary equilibrium (where money prices only depend on the current states) is locally isolated. In contrast, Spear, Srivastava, and Woodford (1990) showed that the "first-" and "second-order" stationary monetary equilibria (where money prices may depend on the past states) are indeterminate. In our paper, the stationarity always refers to the "zero-th order."

${ }^{26}$ The basic idea is as follows. Let $s_{1}:=\arg \min _{s^{\prime} \in S} \omega_{s^{\prime}}^{o}+q_{s^{\prime}}^{*}$ and let $\mathcal{M}_{1}$ be the set of probability measures
} 
to this unique measure (which depends on $s$ because $\mathcal{P}_{s}$ depends on $s$ ) as $\mu_{s}$. The system of inclusions, (4), is then a system of simultaneous equations:

$$
(\forall s \in S) \quad 0=-\sum_{s^{\prime} \in S} q_{s}^{*} u_{1}\left(\omega_{s}^{y}-q_{s}^{*}, \omega_{s^{\prime}}^{o}+q_{s^{\prime}}^{*}\right) \mu_{s s^{\prime}}+\sum_{s^{\prime} \in S} q_{s^{\prime}}^{*} u_{2}\left(\omega_{s}^{y}-q_{s}^{*}, \omega_{s^{\prime}}^{o}+q_{s^{\prime}}^{*}\right) \mu_{s s^{\prime}} .
$$

Any solution to this system is an equilibrium of the economy. Its local nature (including the existence of a continuum of solutions) is the same as the standard stochastic OLG model and is well-known. There is nothing new here and so we have not pursued this line any further. ${ }^{27}$

Next, we consider a partially-insured equilibrium in the following sense. Let $S^{\prime}$ be a subset of $S$ with $\left|S^{\prime}\right| \geq 2$. Consider an equilibrium real money balance $q^{*} \in \Re_{++}^{S}$ such that, for any $s^{\prime}, s^{\prime \prime} \in S^{\prime}, \omega_{s^{\prime}}^{o}+q_{s^{\prime}}^{*}=\omega_{s^{\prime \prime}}^{o}+q_{s^{\prime \prime}}^{*}$. Then, $\mathcal{M}_{s}\left(c_{s}^{M}\left(q^{*}\right)\right)$ includes all the probability measures in $\mathcal{P}_{s}$ that assign the same probability to $S^{\prime}$. However, because $q^{*}$ need not be constant over $S^{\prime}$ (although $\omega^{o}+q^{*}$ must be), the set in (4) is not necessarily a singleton. Therefore, a continuum of solutions to the system of inclusions (4) may arise. To spell out the configuration of endowments that allow such indeterminacy could be very difficult. However, we may choose a continuum of $q^{*}$ 's appropriately so as to keep $\omega^{o}+q^{*}$ constant over some set but to make its (constant) value vary as $q^{*}$ varies. This "endogenized flatness" can be further exploited to show the indeterminacy of fully-insured equilibria.

Recall that a pair of first- and second-period endowments, $\left(\omega^{y}, \omega^{o}\right) \in \Re_{++}^{S} \times \Re_{++}^{S}$, is called an economy. We say that an economy exhibits equilibrium indeterminacy if the stationary monetary equilibrium for it exists and is indeterminate. Furthermore, define the function $f: \Re_{++}^{2} \rightarrow \Re_{++}$ by

$$
f\left(c^{y}, c^{o}\right):=\frac{u_{1}\left(c^{y}, c^{o}\right)}{u_{2}\left(c^{y}, c^{o}\right)}
$$

for each $\left(c^{y}, c^{o}\right) \in \Re_{++}^{2}$, which is the marginal rate of substitution between the first- and secondperiod consumptions. Note that $f$ is continuous because $u$ is continuously differentiable. We can now demonstrate the robust indeterminacy of stationary monetary equilibria.

in $\mathcal{P}_{s}$ that assign the largest probability to $s_{1}$. Then, let $s_{2}:=\arg \min _{s^{\prime} \in S \backslash\left\{s_{1}\right\}} \omega_{s^{\prime}}^{o}+q_{s^{\prime}}^{*}$ and let $\mathcal{M}_{2}$ be the set of probability measures in $\mathcal{M}_{1}$ that assign the largest probability to $s_{2}$. Continuing this process can lead to a single probability measure in $\mathcal{P}_{s}$ that is the unique element of $\mathcal{M}_{s}\left(c_{s}^{M}(q)\right)$. In general, however, the success of this procedure hinges upon the nature of $\mathcal{P}_{s}$. In this sense, the argument of this footnote stands only heuristically. For example, if $\mathcal{P}_{s}$ is characterized by $\varepsilon$-contamination, the above procedure will determine a single probability measure. For the $\varepsilon$-contamination, see Nishimura and Ozaki (2006) and references therein.

${ }^{27}$ As stated in the previous footnote, the system of equations can possibly be a system of inclusions. Because we are concerned with a sufficient condition for multiple equilibria, we can neglect such a situation for our purpose. 
Theorem 2 There exists a nonempty open set $\Omega \subset \Re_{++}^{S} \times \Re_{++}^{S}$ of economies that generate a continuum of fully-insured stationary monetary equilibria, if there are two distinct probability measures on $S$ that belong to $\mathcal{P}_{s}$ for each $s \in S$, and $f\left(\cdot, c^{o}\right)$ is surjective for each $c^{o}>0$.

Therefore, we can find an open set of economies that exhibit equilibrium indeterminacy. More precisely, in the proof of this theorem (see Section 8), we show that the fully-insured secondperiod consumption satisfying Eq. (5) $\left(\bar{c}^{* o}\right)$ becomes indeterminate. This implies that the corresponding equilibrium real money balance $q^{*}$ (calculated using $q_{s}^{*}=\bar{c}^{* o}-\omega_{s}^{o}$ for each $s \in S$ ) is also indeterminate. Hence, we can obtain nominal and real indeterminacy, i.e., indeterminacy of both the equilibrium nominal commodity prices and the equilibrium allocations.

Remark 2 Gottardi (1996) showed that in a more general stochastic OLG model with many agents that have convex preferences and with many securities, a stationary monetary equilibrium generically exists (Theorem 1.i). We can deduce from this result that a stationary monetary equilibrium generically exists in our model with a convex MEU preference. Additionally, Gottardi showed that the equilibrium is locally isolated when the agents' preferences are smooth (Theorem 1.ii). To be more precise, he showed that any open set of economies has its open subset of full Lebesgue measure under convex and smooth preferences and that each element of that subset generates a locally isolated stationary monetary equilibrium. ${ }^{28}$ His observations still hold in our model provided that the agent's belief about the uncertain situation is summarized by a single probability measure. In contrast with his result, however, Theorem 2 shows the existence of some open set of economies that exhibit equilibrium indeterminacy under the MEU preference, which is convex but not smooth. Therefore, Theorem 2 is in stark contrast to previous results under smooth preferences.

We can provide an important property about economies that generate fully-insured stationary monetary equilibria. As argued in the following remark, such economies exhibit aggregate uncertainty.

Remark 3 Let $\omega:=\left(\omega^{y}, \omega^{o}\right) \in \Re_{++}^{S} \times \Re_{++}^{S}$ be an arbitrary economy generating indetermi-

\footnotetext{
${ }^{28}$ Interested readers may refer to Cass, Green and Spear (1992), which also provided the existence and regularity results for the stationary monetary equilibrium in a more complicated stochastic OLG model.
} 
nacy of fully-insured stationary monetary equilibria. Let $\bar{c}^{* o}$ be a second-period consumption corresponding to some fully-insured stationary monetary equilibrium given the economy. We can observe that this economy exhibits aggregate uncertainty. To be more precise, it holds that $\omega_{s}^{y}+\omega_{s}^{o} \neq \omega_{s^{\prime}}^{y}+\omega_{s^{\prime}}^{o}$ for some $s, s^{\prime} \in S$. First, suppose that $\omega_{s}^{y}=\omega_{s^{\prime}}^{y}=: \bar{\omega}^{y}$ and $\omega_{s}^{o}=\omega_{s^{\prime}}^{o}=: \bar{\omega}^{o}$ for each $s, s^{\prime} \in S$. Then, $q_{s}^{*}=\bar{c}^{* o}-\bar{\omega}^{o}=q_{s^{\prime}}^{*}=: \bar{q}^{*}$ for each $s, s^{\prime} \in S$ and Eq.(5) degenerates into $u_{1}\left(\bar{\omega}^{y}-\bar{q}^{*}, \bar{\omega}^{o}+\bar{q}^{*}\right) / u_{2}\left(\bar{\omega}^{y}-\bar{q}^{*}, \bar{\omega}^{o}+\bar{q}^{*}\right)=1$. This characterizes a stationary monetary equilibrium of a deterministic OLG model with the initial endowment $\left(\bar{\omega}^{y}, \bar{\omega}^{o}\right)$. However, we can deduce from Kehoe and Levine (1984, Proposition 3.5) that solutions of this equation must be locally isolated, which contradicts the hypothesis that $\omega$ exhibits indeterminacy. Therefore, there exist some $\tau, \kappa \in S$ such that $\omega_{\tau}^{y} \neq \omega_{\kappa}^{y}$ or $\omega_{\tau}^{o} \neq \omega_{\kappa}^{o}$. Now, suppose that $\omega_{s}^{y}+\omega_{s}^{o}=\omega_{s^{\prime}}^{y}+\omega_{s^{\prime}}^{o}=: \bar{\omega}$ for each $s, s^{\prime} \in S$. Using the previous argument, it holds that $\omega_{\tau}^{o} \neq \omega_{\kappa}^{o}$. Then, Eq.(5) can be rewritten as $\min _{\pi \in \mathcal{P}_{s}} \sum_{s^{\prime} \in S}\left(\bar{c}^{o *}-\omega_{s^{\prime}}^{o}\right) \pi_{s^{\prime}} /\left(\bar{c}^{* o}-\omega_{s}^{o}\right) \leq u_{1}\left(\bar{\omega}-\bar{c}^{* o}, \bar{c}^{* o}\right) / u_{2}\left(\bar{\omega}-\bar{c}^{* o}, \bar{c}^{* o}\right) \leq$ $\max _{\pi \in \mathcal{P}_{s}} \sum_{s^{\prime} \in S}\left(\bar{c}^{o *}-\omega_{s^{\prime}}^{o}\right) \pi_{s^{\prime}} /\left(\bar{c}^{* o}-\omega_{s}^{o}\right)$ for each $s \in S$. However, we can find that there is no constant $u_{1}\left(\bar{\omega}-\bar{c}^{* o}, \bar{c}^{* o}\right) / u_{2}\left(\bar{\omega}-\bar{c}^{* o}, \bar{c}^{* o}\right)$ that satisfies the last inequality for each $s .^{29}$ Therefore, each economy found in Theorem 2 exhibits the aggregate uncertainty.

The last condition in Theorem 2 can be replaced with a more simple condition when the utility function $u$ is additively time-separable. In the rest of this section, we concentrate our attention on the case where $u$ is additively time-separable.

Corollary 2 Suppose that $u$ is time-separable, i.e., there exist some strictly monotone increasing and strictly concave real-valued functions $v_{y}$ and $v_{o}$ on $\Re_{+}$, which are continuously differentiable on the interior of its domain, such that $u\left(c^{y}, c^{o}\right)=v_{y}\left(c^{y}\right)+v_{o}\left(c^{o}\right)$ for each $\left(c^{y}, c^{o}\right) \in \Re_{+} \times \Re_{+}$. Then, there exists a nonempty open set of economies that exhibit equilibrium indeterminacy if $\mathcal{P}_{s}$ has two distinct probability measures for each $s \in S$ and $v_{y}$ satisfies the Inada condition, i.e., $\lim _{c^{y} \downarrow 0} v_{y}^{\prime}\left(c^{y}\right)=+\infty$ and $\lim _{c^{y} \uparrow+\infty} v_{y}^{\prime}\left(c^{y}\right)=0$.

Remark 4 By restricting the preferences to the ones which are convex and smooth, we can find economies that generate a unique stationary monetary equilibrium. Under the assumption of

\footnotetext{
${ }^{29}$ Consider $\bar{s}$ and $\underline{s}$ such that $\omega_{\bar{s}}=\max _{s \in S} \omega_{s}^{o}$ and $\omega_{\underline{s}}=\min _{s \in S} \omega_{s}^{o}$. Because $\pi \gg 0$ for each $\pi \in \mathcal{P}_{s}$, $\bar{c}^{* o}$ should satisfy that $1<\min _{\pi \in \mathcal{P}_{\underline{s}}} \sum_{s^{\prime} \in S}\left(\bar{c}^{o *}-\omega_{s^{\prime}}^{o}\right) \pi_{s^{\prime}} /\left(\bar{c}^{* o}-\omega_{\underline{s}}^{o}\right) \leq u_{1}\left(\bar{\omega}-\bar{c}^{* o}, \bar{c}^{* o}\right) / u_{2}\left(\bar{\omega}-\bar{c}^{* o}, \bar{c}^{* o}\right) \leq$ $\max _{\pi \in \mathcal{P}_{\bar{s}}} \sum_{s^{\prime} \in S}\left(\bar{c}^{o *}-\omega_{s^{\prime}}^{o}\right) \pi_{s^{\prime}} /\left(\bar{c}^{* o}-\omega_{\bar{s}}^{o}\right)<1$. However, this is a contradiction.
} 
additive separability of $u$, Magill and Quinzii (2003, Proposition 1) provide a sufficient condition for the uniqueness of stationary monetary equilibrium. Following their result, if there is a stationary monetary equilibrium under a unique prior, it can be unique when the degree of relative risk aversion of the utility function for the second-period consumption is less than or equal to one. ${ }^{30}$ However, it follows from Corollary 2 that there exists a continuum of stationary monetary equilibria under the MEU preference even when the degree of relative risk aversion of the second-period utility function is less than or equal to one. Therefore, Corollary 2 is a stark contrast to previous results under the smooth and additively time-separable preferences. ${ }^{31}$

In this subsection, we have found the robust indeterminacy of stationary monetary equilibria. This indeterminacy is proved via the "endogenized flatness"; i.e., the fact that we can choose equilibrium real money balances so that the second-period consumptions can be constant over all states.

In the following two sections, we provide further results on the fully-insured stationary monetary equilibria.

\section{Comparative Statics}

This section shows that an increase in ambiguity implies both an increase in the "degree" of indeterminacy and the dilation of the set of economies that exhibit a continuum of fully-insured stationary monetary equilibria. Given an economy $\omega=\left(\omega^{y}, \omega^{o}\right)$ and a transition probability correspondence $\mathcal{P}$, we define the set of second-period consumptions at fully-insured stationary monetary equilibria by $\hat{C}_{\mathcal{P}}^{o}(\omega)$. That is, we let

$$
\hat{C}_{\mathcal{P}}^{o}(\omega):=\left\{\bar{c}^{o} \in \Re_{++} \mid \bar{c}^{o} \text { satisfies Eq.(5) under } \mathcal{P} \text { and } \omega\right\} \text {. }
$$

Note that $\hat{C}_{\mathcal{P}}^{o}(\omega)$ may be empty for some $\omega$. Additionally, define $\hat{\Omega}_{\mathcal{P}}$ by

$$
\hat{\Omega}_{\mathcal{P}}:=\left\{\omega \in \Re_{++}^{S} \times \Re_{++}^{S} \mid \hat{C}_{\mathcal{P}}^{o}(\omega) \neq \emptyset\right\} .
$$

\footnotetext{
${ }^{30}$ This result can be extended to slightly more general preferences. See Ohtaki (2013b).

${ }^{31}$ In the framework of additively time-separable preferences, Manuelli (1990) also provides uniqueness results under an additional assumption that the states' evolution follows an i.i.d. process. He considers a stochastic OLG model with a general state space, and hence, it includes a finite state space case as in our model as a special case. Note that even when the set of priors, $\mathcal{P}_{s}$, is independent of $s$, Corollary 2 still demonstrates the existence of a continuum of stationary monetary equilibria.
} 
Note that $\hat{\Omega}_{\mathcal{P}}$ is the set of economies in which a fully-insured stationary monetary equilibrium exists. Finally, we write $\mathcal{P} \subset \mathcal{Q}$ when $\mathcal{P}_{s} \subset Q_{s}$ for each $s \in S$. In accordance with the notion of Ghirardato and Marinacci (2002), we may say that $\mathcal{Q}$ represents more ambiguity (and more ambiguity aversion given the MEU preference) than $\mathcal{P}$, when $\mathcal{P} \subset \mathcal{Q}$. We can then prove the following theorem.

Theorem 3 Let $\mathcal{P}$ and $\mathcal{Q}$ be two transition probability correspondences such that $\mathcal{P} \subset \mathcal{Q}$. Also assume that $u$ and $\mathcal{P}$ satisfy all the assumptions of Theorem 2 (and hence, $\hat{\Omega}_{\mathcal{P}}$ is nonempty by Theorem 2). Then, (a) $\hat{C}_{\mathcal{P}}^{o}(\omega) \subset \hat{C}_{\mathcal{Q}}^{o}(\omega)$ for any $\omega \in \hat{\Omega}_{\mathcal{P}}$; and $(b) \hat{\Omega}_{\mathcal{P}} \subset \hat{\Omega}_{\mathcal{Q}}$.

Recall that $\hat{\Omega}_{\mathcal{P}}$ and $\hat{C}_{\mathcal{P}}^{o}(\omega)$ are the set of economies that exhibit indeterminacy (of the fullyinsured equilibrium) and the set of second-period consumptions for some fully-insured equilibrium. As mentioned above, the theorem indicates that an increase in uncertainty increases the degree of indeterminacy of the fully-insured stationary monetary equilibrium and, at the same time, expands the set of economies that exhibit a continuum of fully-insured equilibria.

Remark $\mathbf{5}$ The above observation is made by the comparative statics with respect to ambiguity. Following Ghirardate and Marinacci (2002), we define "greater ambiguity" as dilation of the set of priors. As argued in Klibanoff, Marinacci and Mukerji (2005), such a definition of greater ambiguity cannot disentangle an attitude toward ambiguity from ambiguity itself. ${ }^{32}$ In other words, our definition of greater ambiguity involves the effects of both increasing ambiguity and increasing ambiguity aversion. Theorem 3 then states that such a greater ambiguity increases the sizes of both the set of stationary monetary equilibria and the set of economies that exhibit equilibrium indeterminacy. To the best of our knowledge, this paper is the first work that conducts comparative statics with respect to ambiguity in a stochastic OLG model with money.

\section{Optimality}

We have shown the existence of a continuum of stationary monetary equilibria. A question that naturally arises is whether the allocations corresponding to these stationary monetary

\footnotetext{
${ }^{32}$ Klibanoff, Marinacci and Mukerji (2005) attempt to separate these two essentially distinct aspects of the preference. Gajdos, Hayashi, Tallon and Vergnaud (2008) also do so by using "objective" uncertainty.
} 
equilibria are optimal or not. The answer is that they are, in some sense. We begin with some definitions.

Let $S_{0}:=\left\{s_{0}\right\} \cup S$. A stationary feasible allocation is a pair of $c^{y} \in \Re_{++}^{S}$ and $c^{o} \in \Re_{++}^{S_{0} \times S}$ that satisfy

$$
\left(\forall\left(s^{\prime}, s\right) \in S_{0} \times S\right) \quad c_{s}^{y}+c_{s^{\prime} s}^{o}=\omega_{s}^{y}+\omega_{s}^{o}
$$

where $c_{s_{0} s}^{o}$ is the consumption of the initial old, who lives for one period in period 1 and in state $s$. From this equation and the assumption that the endowment depends only on the current state (see the discussion right after the definition of stationary monetary equilibrium in Subsection 3.3 ), it follows that $c_{s^{\prime} s}^{o}$ is independent of $s^{\prime}$. Thus, we define a stationary feasible allocation as $c=\left(c_{s}^{y}, c_{s}^{o}\right)_{s \in S}$. A stationary feasible allocation $b=\left(b_{s}^{y}, b_{s}^{o}\right)_{s \in S}$ is conditionally Pareto superior to a stationary feasible allocation $c=\left(c_{s}^{y}, c_{s}^{o}\right)_{s \in S}$ if

$$
(\forall s \in S) \quad U_{s}\left(b_{s}^{y},\left(b_{s^{\prime}}^{o}\right)_{s^{\prime} \in S}\right) \geq U_{s}\left(c_{s}^{y},\left(c_{s^{\prime}}^{o}\right)_{s^{\prime} \in S}\right) \quad \text { and } \quad b_{s}^{o} \geq c_{s}^{o}
$$

with at least one strict inequality. The latter set of inequalities means that the initial old will not be worse off when moving from $c$ to $b$, where we implicitly assume that the initial old will never be satiated. A stationary feasible allocation $c$ is conditionally Pareto optimal if there is no other stationary feasible allocation that is conditionally Pareto superior to $c$.

We are now ready to state the main result of this section.

Theorem 4 Every stationary monetary equilibrium achieves conditional Pareto optimality.

Theorem 4 claims that even when there exists a continuum of stationary monetary equilibrium allocations because of the MEU preferences, each element of the continuum is conditionally Pareto optimal. ${ }^{33}$ In other words, all equilibria found in Theorem 2 are incompatible with each other in the sense of the Pareto superiority.

Remark 6 When the agents' preferences are smooth, the conditionally Pareto optimal allocations are characterized by the dominant root of the matrix of marginal rates of substitution being less than or equal to one (Aiyagari and Peled, 1991; Chattopadhyay and Gottardi, 1999;

\footnotetext{
${ }^{33}$ Note that the proof of Theorem 4 does not require continuous differentiability of $u$.
} 
Chattopadhyay, 2001; Ohtaki, 2013a, among others). However, as we have already seen it, the MEU preferences are nonsmooth. Therefore, we cannot directly apply the characterization results developed by the studies mentioned above to the current model. Instead of applying the established characterization results, we can more directly demonstrate the optimality of the stationary monetary equilibria by tailoring Sakai's (1988) proof to our model. Sakai demonstrates the conditional Pareto optimality of the stationary monetary equilibrium allocations in a stochastic OLG model with the standard expected utility preferences while his proof does not hinge upon this specific form of preferences.

\section{Lemmas and Proofs}

We first prove that each stationary monetary equilibrium given an economy is characterized by a system of inclusions. To handle this case, we apply the following lemma that was directly adapted from Aubin (1979, p.118, Proposition 6).

Lemma 1 (Aubin, 1979) Let $\Pi$ be a nonempty subset of a metric space and let $\left\{f_{\pi}\right\}_{\pi \in \Pi}$ be a collection of functions from $\Re$ to $\Re$. For each $x \in \Re$, define

$$
g(x):=\inf _{\pi \in \Pi} f_{\pi}(x) \quad \text { and } \quad \mathcal{M}(x):=\left\{\pi \in \Pi \mid g(x)=f_{\pi}(x)\right\}
$$

Let $x \in \Re$. Suppose that (a) $\Pi$ is compact; (b) there exists a neighborhood $X$ of $x$ such that functions $\pi \mapsto f_{\pi}(y)$ are continuous (in the metric topology) for all $y \in X$; and (c) for all $\pi \in \Pi$, $f_{\pi}$ is concave and differentiable. Then, $g$ is differentiable at $x$ both from the left and the right and it holds that

$$
D_{-} g(x)=\max _{\pi \in \mathcal{M}(x)} D f_{\pi}(x) \quad \text { and } \quad D_{+} g(x)=\min _{\pi \in \mathcal{M}(x)} D f_{\pi}(x) .
$$

We can apply this lemma to prove Theorem 1 .

Proof of Theorem 1. Because $U_{\mathcal{P}_{s}}(\cdot)$ is strictly concave, a real number $m \in \Re$ is a solution of the degenerate optimization problem in (2) if and only if

$$
(\forall s \in S) \quad D_{+} V_{s}(m) \leq 0 \leq D_{-} V_{s}(m),
$$


where $D_{-} V_{s}(m)$ and $D_{+} V_{s}(m)$ are the left and right derivatives of $V_{s}(m)$ taken with respect to $m$ (the existence of which is given by Lemma 1). Therefore, we can characterize a stationary equilibrium by a system of inequalities:

$$
(\forall s \in S) \quad D_{+} V_{s}(M) \leq 0 \leq D_{-} V_{s}(M)
$$

Because

$$
\begin{aligned}
& D_{+} V_{s}(m)=\min _{\pi \in \mathcal{M}_{s}\left(c_{s}^{m}\left(q^{*}\right)\right)}\left(-\sum_{s^{\prime} \in S} q_{s}^{*} u_{1}\left(c_{s s^{\prime}}^{m}\left(q^{*}\right)\right) \pi_{s^{\prime}}+\sum_{s^{\prime} \in S} q_{s^{\prime}}^{*} u_{2}\left(c_{s s^{\prime}}^{m}\left(q^{*}\right)\right) \pi_{s^{\prime}}\right), \\
& D_{-} V_{s}(m)=\max _{\pi \in \mathcal{M}_{s}\left(c_{s}^{m}\left(q^{*}\right)\right)}\left(-\sum_{s^{\prime} \in S} q_{s}^{*} u_{1}\left(c_{s s^{\prime}}^{m}\left(q^{*}\right)\right) \pi_{s^{\prime}}+\sum_{s^{\prime} \in S} q_{s^{\prime}}^{*} u_{2}\left(c_{s s^{\prime}}^{m}\left(q^{*}\right)\right) \pi_{s^{\prime}}\right)
\end{aligned}
$$

for all $m \in \Re$ and $\mathcal{M}_{s}\left(c_{s}^{M}\left(q^{*}\right)\right)$ is convex for all $s \in S$, we obtain (4).

We need the following lemma to prove Theorem 2.

Lemma 2 Suppose that there are two distinct probability measures $\mu, \nu \in \Delta_{S}$, such that $\mu, \nu \in$ $\mathcal{P}_{s}$ for each $s \in S$. Then, there exists $\hat{\omega}^{o} \in \Re_{++}^{S}$ such that

$$
\min \left\{\sum_{s^{\prime} \in S} \hat{\omega}_{s^{\prime}}^{o} \pi_{s^{\prime}} \mid \pi \in \mathcal{P}_{\hat{s}}\right\}<\max \left\{\sum_{s^{\prime} \in S} \hat{\omega}_{s^{\prime}}^{o} \pi_{s^{\prime}} \mid \pi \in \mathcal{P}_{\tilde{s}}\right\}
$$

for each $\hat{s}, \tilde{s} \in S$, or equivalently,

$$
\max _{s \in S} \min _{\pi \in \mathcal{P}_{s}} \sum_{s^{\prime} \in S} \hat{\omega}_{s^{\prime}}^{o} \pi_{s^{\prime}}<\min _{s \in S} \max _{\pi \in \mathcal{P}_{s}} \sum_{s^{\prime} \in S} \hat{\omega}_{s^{\prime}}^{o} \pi_{s^{\prime}}
$$

Proof of Lemma 4. Because $\mu$ and $\nu$ are distinct probability measures on $S$, there exists at least one $t \in S$ such that $0 \leq \nu_{t}<\mu_{t}$. Define $\bar{\alpha}>0$ by

$$
\bar{\alpha}:=\frac{\sum_{s^{\prime} \neq t}\left(\nu_{s^{\prime}}-\mu_{s^{\prime}}\right)}{1-\frac{\nu_{t}}{\mu_{t}}} .
$$

Because $\sum_{s^{\prime} \neq t}\left(\nu_{s^{\prime}}-\mu_{s^{\prime}}\right)>0$ and $1-\nu_{t} / \mu_{t}>0$, it follows that $\bar{\alpha}>0$. Therefore, we can arbitrarily choose $\alpha \in(0, \bar{\alpha})$. Now, define $\hat{\omega}^{2} \in \Re_{++}^{S}$ by

$$
\left(\forall s^{\prime} \in S\right) \quad \hat{\omega}_{s^{\prime}}^{o}:= \begin{cases}\frac{\alpha}{\mu_{t}} & \text { if } s^{\prime}=t, \\ 1 & \text { otherwise. }\end{cases}
$$

It follows from $\alpha<\bar{\alpha}$ that

$$
\alpha\left(1-\frac{\nu_{t}}{\mu_{t}}\right)+\sum_{s^{\prime} \neq t}\left(\mu_{s^{\prime}}-\nu_{s^{\prime}}\right)<0,
$$


so we can obtain that

$$
\begin{aligned}
& \sum_{s^{\prime} \in S} \hat{\omega}_{s^{\prime}}^{o} \mu_{s^{\prime}}-\sum_{s^{\prime} \in S} \hat{\omega}_{s^{\prime}}^{o} \nu_{s^{\prime}} \\
= & \left(\alpha+\sum_{s^{\prime} \neq t} \mu_{s^{\prime}}\right)-\left(\alpha \frac{\nu_{t}}{\mu_{t}}+\sum_{s^{\prime} \neq t} \nu_{s^{\prime}}\right) \\
= & \alpha\left(1-\frac{\nu_{t}}{\mu_{t}}\right)+\sum_{s^{\prime} \neq t}\left(\mu_{s^{\prime}}-\nu_{s^{\prime}}\right) \\
< & 0,
\end{aligned}
$$

i.e., $\sum_{s^{\prime} \in S} \hat{\omega}_{s^{\prime}}^{o} \mu_{s^{\prime}}<\sum_{s^{\prime} \in S} \hat{\omega}_{s^{\prime}}^{o} \nu_{s^{\prime}}$. Finally, because $\mu, \nu \in \mathcal{P}_{s}$ for each $s \in S$, it holds that

$$
\min \left\{\sum_{s^{\prime} \in S} \hat{\omega}_{s^{\prime}}^{o} \pi_{s^{\prime}} \mid \pi \in \mathcal{P}_{\hat{s}}\right\} \leq \sum_{s^{\prime} \in S} \hat{\omega}^{o} \mu_{s^{\prime}}<\sum_{s^{\prime} \in S} \hat{\omega}^{o} \nu_{s^{\prime}} \leq \max \left\{\sum_{s^{\prime} \in S} \hat{\omega}_{s^{\prime}}^{o} \pi_{s^{\prime}} \mid \pi \in \mathcal{P}_{\tilde{s}}\right\}
$$

for each $\hat{s}, \tilde{s} \in S$. This completes the proof.

Proof of Theorem 2. Let $\hat{\omega}^{o}$ be an element of $\Re_{++}^{S}$ as described in Lemma 4. That is, $\hat{\omega}^{o}$ satisfies (6). There then exist $m, M \in \Re_{++}$such that

$$
(\forall s \in S) \quad \min _{\pi \in \mathcal{P}_{s}} \sum_{s^{\prime} \in S} \hat{\omega}_{s^{\prime}}^{o} \pi_{s^{\prime}}<m<M<\max _{\pi \in \mathcal{P}_{s}} \sum_{s^{\prime} \in S} \hat{\omega}_{s^{\prime}}^{o} \pi_{s^{\prime}} .
$$

Because $\max _{\pi \in \mathcal{P}_{s}} \sum_{s^{\prime} \in S} \omega_{s^{\prime}}^{o} \pi_{s^{\prime}}$ and $\min _{\pi \in \mathcal{P}_{s}} \sum_{s^{\prime} \in S} \omega_{s^{\prime}}^{o} \pi_{s^{\prime}}$ are continuous in $\omega^{o}$ for each $s \in S$ according to the maximum theorem (Berge, 1963), there exists an open neighborhood, $\Omega_{s}^{o}$, of $\hat{\omega}^{o}$ for each $s \in S$ such that any element of $\Omega_{s}^{o}$ satisfies

$$
\min _{\pi \in \mathcal{P}_{s}} \sum_{s^{\prime} \in S} \omega_{s^{\prime}}^{o} \pi_{s^{\prime}}<m<M<\max _{\pi \in \mathcal{P}_{s}} \sum_{s^{\prime} \in S} \omega_{s^{\prime}}^{o} \pi_{s^{\prime}} .
$$

Define $\Omega^{o}$ by $\Omega^{o}:=\bigcap_{s \in S} \omega_{s}^{o}$. Obviously, $\Omega^{o}$ is open and nonempty because $\hat{\omega}^{o} \in \Omega^{o}$. There then exist $\underline{\omega}_{1}^{o}, \bar{\omega}_{1}^{o}, \ldots, \underline{\omega}_{|S|}^{o}, \bar{\omega}_{|S|}^{o}$ such that $\hat{\omega}^{o} \in\left(\underline{\omega}_{1}^{o}, \bar{\omega}_{1}^{o}\right) \times \cdots\left(\underline{\omega}_{|S|}^{o}, \bar{\omega}_{|S|}^{o}\right) \subset \Omega^{o}$, where we write $S=\{1,2, \ldots,|S|\}$. Let $\hat{c}^{o}$ be any real number such that $\hat{c}^{o}>\max _{s \in S} \bar{\omega}_{s}^{o}$. Then, from the above inequalities, it holds that, for any $\omega^{o} \in\left(\underline{\omega}_{1}^{o}, \bar{\omega}_{1}^{o}\right) \times \cdots\left(\underline{\omega}_{|S|}^{o}, \bar{\omega}_{|S|}^{o}\right)$ and for any $s \in S$,

$$
\hat{c}^{o}-\max _{\pi \in \mathcal{P}_{s}} \sum_{s^{\prime} \in S} \omega_{s^{\prime}}^{o} \pi_{s^{\prime}}<\hat{c}^{o}-M<\hat{c}^{o}-m<\hat{c}^{o}-\min _{\pi \in \mathcal{P}_{s}} \sum_{s^{\prime} \in S} \omega_{s^{\prime}}^{o} \pi_{s^{\prime}} .
$$

Let $s \in S$. Define a function $g: \Re_{++}^{2} \rightarrow \Re$ by

$$
\left(\forall\left(\omega_{s}^{y}, \omega_{s}^{o}\right)\right) \quad g\left(\omega_{s}^{y}, \omega_{s}^{o}\right):=\left(\hat{c}^{o}-\omega_{s}^{o}\right) \frac{u_{1}\left(\omega_{s}^{y}+\omega_{s}^{o}-\hat{c}^{o}, \hat{c}^{o}\right)}{u_{2}\left(\omega_{s}^{y}+\omega_{s}^{o}-\hat{c}^{o}, \hat{c}^{o}\right)} .
$$


By the assumption that $f$ is surjective with respect to its first argument, there exists $\hat{\omega}_{s}^{y} \in \Re_{++}$ such that $g\left(\hat{\omega}_{s}^{y}, \hat{\omega}_{s}^{o}\right) \in\left(\hat{c}^{o}-M, \hat{c}^{o}-m\right)$, where $\hat{\omega}_{s}^{o}$ is found in the first paragraph of this proof. Because $g$ is continuous according to the continuity of $f, g^{-1}\left(\left(\hat{c}^{o}-M, \hat{c}^{o}-m\right)\right)$ is open. Therefore, there exist open sets $\Omega_{s}^{y}$ and $\hat{\Omega}_{s}^{o}$ such that $\hat{\omega}_{s}^{y} \in \Omega_{s}^{y}, \hat{\omega}_{s}^{o} \in \hat{\Omega}_{s}^{o}$ and $\Omega_{s}^{y} \times \hat{\Omega}_{s}^{o} \subset g^{-1}\left(\left(\hat{c}^{o}-M, \hat{c}^{o}-m\right)\right)$. Let $\tilde{\Omega}_{s}^{o}:=\left(\underline{\omega}_{s}^{o}, \bar{\omega}_{s}^{o}\right) \cap \hat{\Omega}_{s}^{o}$. Then, $\tilde{\Omega}_{s}^{o}$ is open and nonempty because $\hat{\omega}_{s}^{o}$ is included in both sets. Finally, define $\Omega$ by $\Omega:=\Omega_{1}^{y} \times \cdots \times \Omega_{|S|}^{y} \times \tilde{\Omega}_{1}^{o} \times \cdots \times \tilde{\Omega}_{|S|}^{o}$.

Let $\left(\omega^{y}, \omega^{o}\right) \in \Omega$. Then, $\hat{c}^{o}$ (found in the first paragraph of this proof) satisfies Eq.(5) with strict inequalities for each $s \in S$. Because $f$ is continuous, there exists an open neighborhood, $\hat{C}_{s}^{o}$, of $\hat{c}^{o}$ such that, for any $c^{o} \in \hat{C}_{s}^{o}$, $c^{o}$ satisfies (5) with strict inequalities. Define $C^{o}$ by $C^{o}:=\bigcap_{s \in S} \hat{C}_{s}^{o}$. This is the desired set and the proof is complete.

Proof of Corollary 2. Under the stated assumptions, the function $f$ will become $f(x, y)=$ $v_{y}^{\prime}(x) / v_{o}^{\prime}(y)$ for each $x$ and $y$. According to the Inada condition on $v_{y}, f(\cdot, y)$ is clearly surjective for each $y$, which completes the proof.

Proof of Theorem 3. (a) Let $\omega \in \hat{\Omega}_{\mathcal{P}}$ and $\bar{c}^{o} \in \hat{C}_{\mathcal{P}}^{o}(\omega)$. These definitions are valid because $\hat{\Omega}_{\mathcal{P}}$ is nonempty owing to our assumption and Theorem 2 , and because $\hat{C}_{\mathcal{P}}^{o}(\omega)$ is nonempty according to the definition of $\hat{\Omega}_{\mathcal{P}}$. Then, for each $s \in S$,

$$
\begin{aligned}
\bar{c}^{o}-\max _{\pi \in \mathcal{Q}_{s}} \sum_{s^{\prime} \in S} \omega_{s^{\prime}}^{2} \pi_{s^{\prime}} & \leq \bar{c}^{o}-\max _{\pi \in \mathcal{P}_{s}} \sum_{s^{\prime} \in S} \omega_{s^{\prime}}^{2} \pi_{s^{\prime}} \\
& \leq\left(\bar{c}^{o}-\omega_{s}^{o}\right) f\left(\omega_{s}^{y}+\omega_{s}^{o}-d, d\right) \\
& \leq \bar{c}^{o}-\min _{\pi \in \mathcal{P}_{s}} \sum_{s^{\prime} \in S} \omega_{s^{\prime}}^{2} \pi_{s^{\prime}} \\
& \leq \bar{c}^{o}-\min _{\pi \in \mathcal{Q}_{s}} \sum_{s^{\prime} \in S} \omega_{s^{\prime}}^{2} \pi_{s^{\prime}} .
\end{aligned}
$$

Here, the first and the last inequalities hold because $\mathcal{P}_{s} \subset \mathcal{Q}_{s}$, and the second and third inequalities hold because of the assumption that $d \in \hat{C}_{\mathcal{P}}^{o}(\omega)$. Therefore, $d \in \hat{C}_{Q}^{o}(\omega)$, and hence, $\hat{C}_{\mathcal{P}}^{o}(\omega) \subset \hat{C}_{\mathcal{P}}^{o}(\omega)$.

(b) Let $\omega \in \hat{\Omega}_{\mathcal{P}}$. Then, $\hat{C}_{\mathcal{P}}^{o}(\omega)$ is nonempty, and $\hat{C}_{\mathcal{Q}}^{o}(\omega)$ is also nonempty because $\hat{C}_{\mathcal{P}}^{o}(\omega) \subset$ $\hat{C}_{Q}^{o}(\omega)$ according to $(\mathrm{a})$. Therefore, $\omega \in \hat{\Omega}_{Q}$, which completes the proof. 
Proof of Theorem 4. Sakai (1988) proved that if $U^{s}: \Re_{++} \times \Re_{++}^{S} \rightarrow \Re$ is increasing and strictly quasi-concave for all $s \in S$, then, for any stationary feasible allocation $c$ corresponding to a stationary monetary equilibrium, there exists no other stationary feasible allocation $b$ that is conditionally Pareto superior to $c$. (Sakai (1988) assumed that $U^{s}$ is represented by an expected utility function with a utility index that satisfies some regularity conditions. However, his proof only requires that $U^{s}$ should be increasing and strictly quasi-concave.) Clearly, $U^{s}$ is increasing. Furthermore, because $U^{s}(\cdot)$ is strictly concave, it is strictly quasi-concave. ${ }^{34}$ Therefore, the proof is complete.

\section{Acknowledgement}

The second author's work was supported by JSPS KAKENHI Grant Number 23530226. This work was also supported by the Grant-in-Aid for the Global COE Program "Raising Market Quality-Integrated Design of Market Infrastructure" from MEXT. Both authors thank the two anonymous referees and participants at numerous seminars for their helpful comments.

\section{References}

Aiyagari, S.R. and D. Peled (1991): "Dominant root characterization of Pareto optimality and the existence of optimal equilibria in stochastic overlapping generations models," Journal of Economic Theory 54, 69-83.

Alon, S. and D. Schmeidler (2014): "Purely subjective maxmin expected utility," Journal of Economic Theory 152, 382-412.

Anscombe, F.J. and R.J. Aumann (1963): "A definition of subjective probability," Annals of Mathematical Statistics 34, 199-205.

Aubin, J.P. (1979): Mathematical Methods of Game and Economic Theory, North-Holland, Amsterdam.

Billot, A., A. Chateauneuf, I. Gilboa, and J.-M. Tallon (2000): "Sharing beliefs: Between agreeing and disagreeing," Econometrica 68, 685-694.

Casadesus-Masanell, R., Klibanoff, P. and E. Ozdenoren (2000): "Maxmin Expected Utility over Savage Acts with a Set of Priors," Journal of Economic Theory 92, 35-65.

${ }^{34}$ See, for example, Theorem 1.E.1(i) of Takayama (1974). 
Cass, D, R.C. Green, and S.E. Spear (1992): "Stationary equilibria with incomplete markets and overlapping generations," International Economic Review 33, 495-512.

Chateauneuf, A., R.-A. Dana, and J.-M Tallon (2000): "Risk sharing rules and equilibria with non-additive expected utilities," Journal of Mathematical Economics 61(4), 953-957.

Chattopadhyay, S. (2001): "The unit root property and optimality: a simple proof," Journal of Mathematical Economics 36, 151-159.

Chattopadhyay, S. and P. Gottardi (1999): "Stochastic OLG models, market structure, and optimality," Journal of Economic Theory 89, 21-67.

Dana, R.-A. (2004): "Ambiguity, uncertainty aversion and equilibrium welfare," Economic Theory $23,569-587$.

Demange, G. and G. Laroque (1999): "Social security and demographic shocks," Econometrica 67, 527-542.

Diamond, P.A. (1965): "National debt in a neoclassical growth model," American Economic Review 55, 1126-1150.

Dow, J. and S.R.C. Werlang (1992): "Uncertainty aversion, risk aversion, and the optimal choice of portfolio," Econometrica 60(1), 197-204.

Ellsberg, D. (1961): "Risk, ambiguity, and the Savage axioms," Quarterly Journal of Economics 75, 643-669.

Epstein, L.G. and M. Schneider (2010): "Ambiguity and Asset Markets," Annual Review of Financial Economics 2, 315-346.

Epstein, L.G. and T. Wang (1994): "Intertemporal asset pricing under Knightian uncertainty," Econometrica 62, 283-322.

Epstein, L.G. and T. Wang (1995): "Uncertainty, risk-neutral measures and security price booms and crashes," Journal of Economic Theory 67, 40-82.

Etner, J., M. Jeleva, and J.-M. Tallon (2012): "Decision theory under ambiguity," Journal of Economic Surveys 26, 234-270.

Faro, J.H. (2013): "Cobb-Douglas preferences under uncertainty," Economic Theory 54, 273285. 
Fukuda, S.-i. (2008): "Knightian uncertainty and poverty trap in a model of economic growth," Review of Economic Dynamics 11, 652-663.

Gajdos, T, T. Hayashi, J.-M. Tallon, and J.-C. Vergnaud (2008) "Attitude toward imprecise information," Journal of Economic Theory 140, 27-65.

Gilboa, I. and M. Marinacci (2013): "Ambiguity and the Bayesian Paradigm," in: D. Acemoglu, M. Arellano, and E. Dekel ed. Advances in Economics and Econometrics Tenth World Congress vol.1, Cambridge University, Cambridge, 179-242

Gilboa, I. and D. Schmeidler (1989): "Maxmin expected utility with non-unique prior," Journal of Mathematical Economics 18, 141-153.

Ghirardato, P. and M. Marinacci (2002): "Ambiguity made precise," Journal of Economic Theory 102, 251-289.

Gottardi, P. (1996): "Stationary monetary equilibria in overlapping generations models with incomplete markets," Journal of Economic Theory 71, 75-89.

Guidolin, M. and F. Rinaldi (2013): "Ambiguity in asset pricing and portfolio choice: a review of the literature," Theory and Decision 74, 183-217.

Kehoe, T.J. and D.K. Levine (1984) "Regularity in overlapping generations exchange economies," Journal of Mathematical Economics 13, 69-93.

Klibanoff, P., M. Marinacci, and S. Mukerji (2005): "A smooth model of decision making under ambiguity," Econometrica 73, 1849-1892.

Knight, F. (1921): Risk, Uncertainty and Profit, Houghton Mifflin, Boston.

Labadie, P. (2004): "Aggregate risk sharing and equivalent financial mechanisms in an endowment economy of incomplete participation," Economic Theory 27, 789-809.

Lucas, R.E., Jr. (1978): "Asset prices in an exchange economy," Econometrica 46, 1429-1445.

Maccheroni, F., M. Marinacci, and A. Rustihici (2006): "Ambiguity aversion, robustness, and the variational representation of preferences," Econometrica 74, 1447-1498.

Magill, M. and M. Quinzii (2003): "Indeterminacy of equilibrium in stochastic OLG models," Economic Theory 21, 435-454.

Mandler, M. (2013): "Endogenous indeterminacy and volatility of asset prices under ambiguity," Theoretical Economics 8, 729-750. 
Manuelli, R. (1990): "Existence and optimality of currency equilibrium in stochastic overlapping generations models: The pure endowment case," Journal of Economic Theory 51, 268-294.

Mukerji, S. and J.-M. Tallon (2001): "Ambiguity aversion and incompleteness of financial markets," Review of Economic Studies 68(4), 883-904.

Mukerji, S. and J.-M. Tallon (2004): "An overview of economic applications of David Schmeidler's models of decision making under uncertainty," in Gilboa, I. ed. Uncertainty in Economic Theory, Routledge, New York.

Nishimura, K.G. and H. Ozaki (2006): "An axiomatic approach to $\varepsilon$-contamination," Economic Theory 27, 333-340.

Ohtaki, E. (2011): "A note on the existence of monetary equilibrium in a stochastic OLG model with a finite state space," Economics Bulletin 31, 485-492.

Ohtaki, E. (2013a): "Golden rule optimality in stochastic OLG economies," Mathematical Social Sciences 65, 60-66.

Ohtaki, E. (2013b): "A note on the existence and uniqueness of stationary monetary equilibrium in a stochastic OLG model," Macroeconomic Dynamics, forthcoming (DOI: http://dx.doi.org/10.1017/S1365100513000515).

Ohtaki, E. (2014): "Tractable graphical device for analyzing stationary stocastic OLG Economies," Journal of Macroeconomics 40, 16-26.

Ohtaki, E. and H. Ozaki (2013): "Optimality in a stochastic OLG model with ambiguity," Tokyo Center for Economic Research Paper No. E-69. Available at SSRN: http://ssrn.com/abstract $=2377585$.

Rigotti, L. and C. Shannon (2012): "Sharing risk and ambiguity," Journal of Economic Theory 147, 2028-2039.

Rinaldi, F. (2009) "Endogenous incompleteness of financial markets: The role of ambiguity and ambiguity aversion," Journal of Mathematical Economics 45, 880-901.

Sakai, Y. (1988): "Conditional Pareto optimality of stationary equilibrium in a stochastic overlapping generations Model," Journal of Economic Theory 44, 209-213.

Savage, L.J. (1954): The Foundations of Statistics, John Wiley, New York (2nd ed., 1972, Dover, New York). 
Schmeidler, D. (1982): "Subjective probability without additivity" (Temporary Title), Working Paper, The Foerder Institute for Economic Research, Tel Aviv University.

Schmeidler, D. (1989): "Subjective probability and expected utility without additivity," Econometrica $\mathbf{5 7}, \mathbf{5 7 1 - 5 8 7}$. (Its working paper is first published in 1982)

Spear, S.E., S. Srivastava, and M. Woodford (1990): "Indeterminacy of stationary equilibrium in stochastic overlapping generations models," Journal of Economic Theory 50, 265-284.

Takayama, A. (1974): Mathematical Economics, The Dryden Press, Hinsdale. IL.

Tallon, J.-M. (1998): "Do sunspots matter when agents are Choquet-expected-utility maxmizers?," Journal of Economic Dynamics and Control 22, 357-368. 